\title{
Black-hole hair loss: learning about binary progenitors from ringdown signals
}

\author{
Ioannis Kamaretsos, ${ }^{1}$ Mark Hannam, ${ }^{1}$ Sascha Husa,${ }^{2}$ and B.S. Sathyaprakash ${ }^{1}$ \\ ${ }^{1}$ School of Physics and Astronomy, Cardiff University, Queens Building, CF24 3AA, Cardiff, United Kingdom \\ ${ }^{2}$ Departament de Física, Universitat de les Illes Balears, Crta. Valldemossa km 7.5, E-07122 Palma, Spain
}

\begin{abstract}
Perturbed Kerr black holes emit gravitational radiation, which (for the practical purposes of gravitational-wave astronomy) consists of a superposition of damped sinusoids termed quasi-normal modes. The frequencies and time-constants of the modes depend only on the mass and spin of the black hole - a consequence of the no-hair theorem. It has been proposed that a measurement of two or more quasi-normal modes could be used to confirm that the source is a black hole and to test if general relativity continues to hold in ultra-strong gravitational fields. In this paper we propose a practical approach to testing general relativity with quasi-normal modes. We will also argue that the relative amplitudes of the various quasi-normal modes encode important information about the origin of the perturbation that caused them. This helps in inferring the nature of the perturbation from an observation of the emitted quasi-normal modes. In particular, we will show that the relative amplitudes of the different quasi-normal modes emitted in the process of the merger of a pair of nonspinning black holes can be used to measure the component masses of the progenitor binary.
\end{abstract}

PACS numbers: 04.30.Db, 04.25.Nx, 04.80.Nn, 95.55.Ym

\section{INTRODUCTION}

The black hole no-hair theorem states that a charged, stationary and axially symmetric black hole with an event horizon of spherical topology can be described by the Kerr-Newman geometry [1, 2], where it is characterized by just three quantities - its mass, spin and electric charge [3 7]. Astrophysical black holes are believed not to have any charge and so are described by the Kerr geometry [1], characterized by just their mass and spin angular momentum. An important consequence of the no-hair theorem concerns the behaviour of Kerr black holes when subjected to an external perturbation. There is strong evidence that black holes are stable against such perturbations [8 14] (see, however, Ref. 15]). Perturbed black holes regain their axisymmetric configuration by emitting gravitational radiation. The radiation observed by a detector takes the form

$$
h(t)=\sum_{\ell, m, n} A_{\ell m n} e^{-t / \tau_{\ell m n}} \cos \left(\omega_{\ell m n} t+\phi_{\ell m n}\right)
$$

and it consists of a superposition of quasi-normal modes (QNMs) with characteristic mode frequencies $\omega_{\ell m n}$ and time-constants $\tau_{\ell m n}$. Here $\ell=2, \ldots$, and $m=-\ell, \ldots, \ell$, are the spheroidal harmonic indices and $n$ is an index corresponding to the overtones of each mode. The amplitudes $A_{\ell m n}$ depend on the relative orientation of the detector and the black hole as well as the nature of the perturbing agent and $\phi_{\ell m n}$ are constants defining the initial phase of the various modes 1 . One of the goals of this paper is to determine the amplitudes of the most significant modes excited during the merger of a pair of black holes.

\footnotetext{
${ }^{1}$ For a recent review on black hole quasi-normal modes see Ref. 16]
}

The mode frequencies and time-constants of a black hole of mass $M$ are given by the general expressions

$$
\omega_{\ell m n}=\frac{F_{\ell m n}(j)}{M}, \quad \tau_{\ell m n}=M G_{\ell m n}(j)
$$

where $F_{\ell m n}(j)$ and $G_{\ell m n}(j)$ are functions of the dimensionless black hole spin magnitude, or Kerr parameter, $j$. All mode frequencies and time-constants then depend only on the mass $M$ and the spin magnitude $j$ of the black hole and no other parameter - a consequence of the no-hair theorem. Several authors have noted that this aspect of the no-hair theorem could be used to test if massive compact objects at galactic cores are actually rotating black holes described by the Kerr metric of general relativity 17-19; alternatively, it could be used as a strong field test of general relativity itself [17].

The key idea behind the proposed tests is the following: If one can reliably decompose the observed gravitational radiation from a ringing black hole into a superposition of different modes, then the frequencies and time-constants of each of the modes could be used to infer the mass and spin of the black hole. If the object is truly a black hole, then the masses and spins obtained from the different modes should all be consistent within the measurement errors. Inconsistencies in the values of the masses and spins inferred from different modes would be an indication of the failure of general relativity or that the radiation was emitted from an object that is not a black hole. If a merging binary does not lead to a black hole then the inspiral phase may not result in a superposition of QNMs that can be characterized by just two parameters. Such signatures could be inferred by a model-independent analysis of the data, e.g., a time-frequency transform, or by assessing the posterior probability for alternative models.

In this paper we estimate the relative amplitudes $A_{\ell m n}$ of the various modes by fitting a superposition of QNMs to the radiation emitted by a merging black-hole binary 
obtained from numerical-relativity simulations. These simulations involve the 'simple' case of initially nonspinning black holes in quasi-circular orbits and different mass ratios of the binary, in the range 1:1 to 1:11. Analytical fits of the amplitudes so obtained are extrapolated to mass ratios of up to 25 , so as to study a variety of different systems. The validity of our extrapolation can only be confirmed by future numerical simulations of binary black holes with such large mass ratios.

From the analytical fits we construct a model waveform and calculate the signal-to-noise ratios (SNRs) in different modes during the merger of supermassive blackhole binaries of total mass in the range $\sim 10^{6}-10^{8} M_{\odot}$ observed with the Laser Interferometer Space Antenna (LISA) 20 and of intermediate-mass black-hole binaries of total mass in the range $\sim 100-10^{3} M_{\odot}$ observed with the Einstein Telescope (ET) 21] and advanced configuration of the Laser Interferometer GravitationalWave Observatory (aLIGO) 22 25]. The response of a gravitational-wave detector is, of course, not separately sensitive to the different modes but to only their superposition. However, it should be possible to measure the relative strengths of the different modes by fitting a generic model to the observed data. We will pursue this latter approach in a forthcoming publication and restrict ourselves to a theoretical study of the relative importance of different modes.

We find that over most of the parameter space explored, the modes with indices $(\ell, m, n)=$ $(2,2,0),(3,3,0),(2,1,0)$ and $(4,4,0)$ have SNRs for the ringdown phase larger than 500 in LISA provided the source is within a red-shift of $z=1$ and larger than 50 in ET provided the source is within a distance of 1 Gpc. For aLIGO, the SNRs in $(2,2,0)$ and $(3,3,0)$ modes are larger than 10 in a significant region of the parameter space. However, other sub-dominant modes will not be visible in aLIGO when the source is at a distance of $1 \mathrm{Gpc}$ or greater. In all cases black hole ringdown signals that result from equal-mass binaries can have far larger SNRs. The distance reach of LISA and ET to ringdown signals is large enough that one can expect a few events per year with quite a large ( $\gtrsim 100)$ SNR [26, 27]. One can, therefore, expect that future observations of black hole mergers will provide an excellent opportunity to test GR using several different QNMs.

We will present a specific implementation of the test of the no-hair theorem and discuss a minimal and a maximal set of parameters that could be used to carry out such a test. The chief result of this paper is that the relative amplitudes of the modes depend on the mass ratio $q$ of the

\footnotetext{
2 Recently, the National Aeronautics an Space Administration in the United States opted out of the LISA mission. However, European Space Agency is pursuing an alternative that is similar in scope to LISA and we believe studying what science LISA could deliver is still very relevant.
}

progenitor binary and that by measuring the relative amplitudes, in addition to the frequency and time-constant, it should be possible to measure the component masses of the binary that led to the QNMs.

The rest of this paper is organized as follows. Section II is devoted to a discussion of the numerical relativity simulations used in this work, focusing on their accuracy, so as to give an idea of how reliable are our estimates of the relative amplitudes of different modes. The waveform model used in this study is given in Section III, stating the conventions and assumptions made in constructing the model. Section IV] constructs the amplitudes of the various modes in the ringdown signal using numerical simulations of Section II Section IV also deals with different options for identifying the ringdown phase, the method that was actually followed, the connection between the mode amplitudes and the mass ratio of the binary from which the black hole results and how this information was included in the signal model. In Section $\mathrm{V}$, we discuss the detectability of the various modes with aLIGO, ET and LISA, and possible astrophysical information we can glean from such observations. Sections VI and VII present the results from a covariance matrix analysis of how well we are able to measure the parameters of the ringing black hole and the progenitor binary. In Section VIII, we propose a practical test of the no-hair theorem, making several remarks on which modes and parameters we could use for such a test. We make concluding remarks and outlook for further work in Section IX. We use a system of units in which the Newton's constant and the speed of light are both set to unity, $c=G=1$.

\section{NUMERICAL SIMULATIONS OF MERGER AND RINGDOWN SIGNALS}

In this section, we shall briefly discuss how the numerical simulations were performed. We used the BAM code 28, 29. The code starts with black-hole-binary puncture initial data [30, 31] generated using a pseudospectral elliptic solver [32], and evolves them with the $\chi$-variant of the moving-puncture 3335 version of the BSSN [36, 37, formulation of the 3+1 Einstein evolution equations. We estimate initial momenta for loweccentricity inspiral using the post-Newtonian methods outlined in [38, 39]. Spatial finite-difference derivatives are sixth-order accurate in the bulk [29], Kreiss-Oliger dissipation terms converge at fifth order and a fourthorder Runge-Kutta algorithm is used for the time evolution. Time interpolation in the Berger-Oliger-like adaptive mesh refinement algorithm converges at second order accuracy. In the limit of infinite resolution the code is thus expected to converge with second order accuracy. However, in the regime of currently feasible simulations, the spatial finite differencing error dominates by far. Artificial dissipation has no measurable effect on the phase accuracy of the waves. For well-resolved simulations we 
thus find sixth-order accuracy, as expected.

The gravitational waves emitted by the binary are calculated from the Newman-Penrose scalar $\Psi_{4}$, extracted at a distance $D_{\mathrm{L}}$ from the source. The details of our implementation of this procedure are given in Ref. [28]. Here we recall those details that are important to this paper. The quantity $D_{\mathrm{L}} \Psi_{4}$ is decomposed into spinweighted spherical harmonics, and related to the GW strain as

$$
\begin{aligned}
D_{\mathrm{L}} \Psi_{4} & =D_{\mathrm{L}}\left(\ddot{h}_{+}-i \ddot{h}_{\times}\right) \\
& =\sum_{\ell, m} \Psi_{4, \ell m-2} Y^{\ell m}(\iota, \phi) .
\end{aligned}
$$

To calculate the radiated power, or luminosity, we require $\dot{h}$, which can be obtained by one time-integration of the spherical-harmonic coefficients $\Psi_{4, \ell m}$. In principle we need to fix only one constant of integration to obtain $\dot{h}$ from $\Psi_{4}$, but in practice the integration is contaminated by numerical noise, see e.g., [40 42. As part of our analysis of the data, we produced both $h$ and $\dot{h}$, by (a) fixing the integration constants to ensure that $h$ oscillates around zero, and rings down to zero, (b) removing low-and high-frequency noise via FFTs, and (c) removing further spurious noise effects by subtracting low-order polynomial fits through the strain.

We use results from simulations of non-spinning binaries with mass ratios $q=\{1,2,3,4\}$ that were previously presented in Refs. 39, 43, and an additional simulation of a $q=11$ binary that was carried out as a part of this study.

Detailed error analyses for the first four simulations were presented in Refs. 39, 43, although those works focused on the inspiral phase, while here we focus on the ringdown phase. We find that for the $q=\{1,2,3,4\}$ simulations, the error in the amplitude of the ringdown signal is dominated by the error due to wave extraction at a finite distance from the source. The wave extraction was performed at $R_{\mathrm{ex}}=70 \mathrm{M}$ for the data used in this paper. Waves were extracted at larger radii $(80 M$ and $90 M$ ), but the numerical resolution was lower at these radii and numerical errors began to dominate the uncertainty of the ringdown waveform in the subdominant modes. We also found that the ratios of the waveform luminosities were remarkably robust with respect to the wave-extraction radius, and for these the errors are even lower; a similar effect was found in Ref. [44, where the amplitude ratios between harmonics also played a major role in the study.

The new $q=11$ simulation included only two orbits of inspiral before merger, and was produced primarily to calculate the ringdown signal. The sizes of the meshrefinement levels were varied to optimize both memory usage and numerical accuracy of the wave extraction, which was now performed at $R_{\mathrm{ex}}=100 \mathrm{M}$. Three simulations were performed to validate the accuracy of the results; the resolutions at the wave extraction sphere were $\{0.533,0.427,0.356\} / M$, and the finest resolution at each black aim to capture the inspiral GW phase with high accuracy; our focus was on the accuracy of the ringdown. Here the amplitude accuracy of all the modes we consider in this paper was within $0.5 \%$, and this uncertainty was dominated by the error due to extraction at a finite distance from the source.

The spin of the final black hole is $0.25 \pm 0.01$, and $(0.3 \pm$ $0.01) \%$ of the energy of the system is radiated during the last two orbits and merger and ringdown. The final black hole recoils by $55 \pm 5 \mathrm{~km} / \mathrm{s}$. The large uncertainty in the recoil is due mostly to having the waveform from only a small number of orbits before merger, which makes it difficult to remove the oscillatory inspiral recoil from the results, as was done in Ref. [39]. Our results for this system are consistent with previous simulations of $q=10$ binaries [45 47.

\section{ANTENNA RESPONSE TO A RINGDOWN SIGNAL}

Quasi-normal modes are transients that live for a very short duration in the detector band: In the case of intermediate-mass black holes that could be observed in $\mathrm{ET}$, the time constant is at best about $60 \mathrm{~ms}$ (for a BH of mass $10^{3} M_{\odot}$ and spin $j=0.7$ ), while for supermassive black holes that could be observed in LISA the longest time constant is about $100 \mathrm{~min}$ (for a $\mathrm{BH}$ of mass $10^{8} M_{\odot}$ and spin $j=0.7$ ) (see Table IV). Consequently, it is not necessary to consider the motion of LISA or ET during the observation of a quasi-normal mode, at least not in the current evaluation of what science one might extract from their observation.

Let us consider the response of an interferometric detector to a ringdown signal. We assume that the radiation is incident from a direction $(\theta, \varphi)$ with respect to, say, a geo-centric coordinate system. Let $\left(\mathbf{e}_{x}^{R}, \mathbf{e}_{y}^{R}, \mathbf{e}_{z}^{R}\right)$ be a set of orthonormal vectors representing a coordinate frame in which the ringdown modes take the transversetraceless form; that is, the metric perturbation $h_{i j}$ due to the ringdown modes can be written in this frame as

$$
h_{i j}=h_{+} e_{+}^{i j}+h_{\times} e_{\times}^{i j}
$$

where $h_{+}$and $h_{\times}$are the plus and cross polarizations, whose explicit expressions for a ringdown signal will be given below, and $\mathbf{e}_{+, \times}$are the polarization tensors given by

$$
\begin{aligned}
& \mathbf{e}_{+}=\mathbf{e}_{x}^{R} \otimes \mathbf{e}_{x}^{R}-\mathbf{e}_{y}^{R} \otimes \mathbf{e}_{y}^{R}, \\
& \mathbf{e}_{\times}=\mathbf{e}_{x}^{R} \otimes \mathbf{e}_{y}^{R}+\mathbf{e}_{y}^{R} \otimes \mathbf{e}_{x}^{R} .
\end{aligned}
$$

The response $h^{A}(t)$ of an interferometer, labelled by $A$, can be written as:

$$
h^{A}(t)=F_{+}^{A}(\theta, \varphi, \psi) h_{+}(t)+F_{\times}^{A}(\theta, \varphi, \psi) h_{\times}(t) .
$$

Here $\psi$ is the polarization angle,

$$
\cos \psi=\mathbf{e}_{\theta} \cdot \mathbf{e}_{x}^{R},
$$


and $F_{+, \times}^{A}(\theta, \varphi, \psi)$ are the antenna pattern functions of the detector given by,

$$
F_{+}^{A}=\mathbf{D}_{i j}^{A} \mathbf{e}_{+}^{i j}, \quad F_{\times}^{A}=\mathbf{D}_{i j}^{A} \mathbf{e}_{\times}^{i j},
$$

where $\mathbf{D}^{A}$ is the detector tensor. If $\mathbf{e}_{1,2}^{A}$ are unit vectors (not necessarily orthogonal to each other) along the two arms of an interferometer, then the detector tensor is given by

$$
\mathbf{D}^{A}=\mathbf{e}_{1}^{A} \otimes \mathbf{e}_{1}^{A}-\mathbf{e}_{2}^{A} \otimes \mathbf{e}_{2}^{A} .
$$

For our purposes it is most useful to express the radiation from a source in the source frame, in terms of its expansion in spin-weighted spherical harmonics of weight -2 , namely ${ }_{-2} Y^{\ell m}$ :

$$
h_{+}-i h_{\times}=\sum_{\ell=2}^{\infty} \sum_{m=-\ell}^{\ell} h_{-2}^{\ell m} Y^{\ell m}(\iota, \phi) .
$$

Here, $(\iota, \phi)$ refer to the co-latitude and the azimuth angle at which the radiation is emitted from the source; $\iota$ is also the angle between the line-of-sight and the orbital (spin) angular momentum of the binary (black hole). The complex coefficients $h^{\ell m}$ in the expansion are referred to as $\ell m$ modes. Explicit expressions for the first few modes in the post-Newtonian approximation for the inspiral phase of a binary's evolution can be found in Kidder [48. It is useful to write the modes explicitly in terms of their real and imaginary parts

$$
h^{\ell m}=A_{\ell m} e^{-i \Phi_{\ell m}}=h_{+}^{\ell m}-i h_{\times}^{\ell m} .
$$

This helps in extracting the amplitude and phase of each mode in terms of its plus and cross modes obtained in numerical simulations:

$$
A_{\ell m}=\sqrt{\left(h_{+}^{\ell m}\right)^{2}+\left(h_{\times}^{\ell m}\right)^{2}}, \quad \Phi_{\ell m}=\tan ^{-1}\left[-\frac{h_{\times}^{\ell m}}{h_{+}^{\ell m}}\right] .
$$

Noting that ${ }_{-2} Y^{\ell m}(\iota, \phi)={ }_{-2} Y^{\ell m}(\iota, 0) e^{i m \phi}$, we can rearrange the sums in Eq. (12) using Eq. 114 to get [40]

$$
\begin{aligned}
& h_{+}=\sum_{\ell, m>0} A_{\ell m} Y_{+}^{\ell m} \cos \left(\Phi_{\ell m}-m \phi\right), \\
& h_{\times}=-\sum_{\ell, m>0} A_{\ell m} Y_{\times}^{\ell m} \sin \left(\Phi_{\ell m}-m \phi\right),
\end{aligned}
$$

where we have dropped the "memory-effect" $m=0$ terms, for which the amplitude is low (see, e.g., a recent numerical study [49]). Note that while these modes are non-oscillatory during inspiral, they do exhibit ringdown, which has been studied in some detail with numerical codes in axial symmetry, where they are the only nonzero modes (see, e.g., [50]). In the above expressions, the angular functions $Y_{+, \times}^{\ell m}(\iota)$ are the following combinations of the spin-weighted spherical harmonics:

$$
\begin{aligned}
& Y_{+}^{\ell m}(\iota) \equiv{ }_{-2} Y^{\ell m}(\iota, 0)+(-1)^{\ell}{ }_{-2} Y^{\ell-m}(\iota, 0), \\
& Y_{\times}^{\ell m}(\iota) \equiv{ }_{-2} Y^{\ell m}(\iota, 0)-(-1)^{\ell}{ }_{-2} Y^{\ell-m}(\iota, 0) .
\end{aligned}
$$

For the inspiral phase of a binary when the two compact bodies are widely separated, post-Newtonian $(\mathrm{PN})$ approximation gives the amplitudes $A_{\ell m}(t)$ and phases $\Phi_{\ell m}(t)$ as expansions in $v / c$, where $v$ is the velocity of the bodies (see, e.g., 48]). Numerical relativity simulations can be used to extract them when the $\mathrm{PN}$ approximation breaks down. In the case of perturbed black holes, which a binary will result in, black hole perturbation theory predicts that the modes are damped sinusoids with their amplitudes and phases given by:

$$
A_{\ell m}=\frac{\alpha_{\ell m} M}{D_{\mathrm{L}}} e^{-t / \tau_{\ell m}}, \quad \Phi_{\ell m}(t)=\omega_{\ell m} t
$$

where $M$ is the mass of the black hole and $D_{\mathrm{L}}$ is its luminosity distance from Earth. Time-constants $\tau_{\ell m}$ and frequencies $\omega_{\ell m}$ can be computed from black hole perturbation theory (see, e.g., Ref. [18] for a recent comprehensive listing of frequencies and time-constants). However, the amplitudes $\alpha_{\ell m}$ depend on the nature of the perturbation and are not analytically accessible in the case of black holes that form from the coalescence of a binary. We shall "measure" them later in this paper using results of our numerical simulations.

Using the above equations, the output of the numerical simulations for the plus and cross polarizations corresponds to the following expressions:

$$
\begin{aligned}
h_{+}(t) & =\sum_{\ell, m>0} \frac{\alpha_{\ell m} M}{D_{\mathrm{L}}} Y_{+}^{\ell m}(\iota) e^{-t / \tau_{\ell m}} \cos \left(\omega_{\ell m} t-m \phi\right), \\
h_{\times}(t) & =\sum_{\ell, m>0} \frac{\alpha_{\ell m} M}{D_{\mathrm{L}}} Y_{\times}^{\ell m}(\iota) e^{-t / \tau_{\ell m}} \sin \left(\omega_{\ell m} t-m \phi\right) .
\end{aligned}
$$

We have dropped the overtone index $n$ from all the relevant quantities (amplitudes, frequencies and timeconstants) of quasi-normal modes, as we are assuming that higher (i.e., $n>0$ ) overtones, quickly become negligible in amplitude, compared to the fundamental $n=0$ overtone. Only the fundamental $n=0$ overtone of the various modes is considered in this paper.

The amplitudes $A_{\ell m}$ of the various modes depend on the nature of the perturbation. For ringdowns resulting from the merger of two non-spinning black holes, $A_{\ell m}$ depend on the mass $M$ of the final black hole and mass ratio $q=m_{1} / m_{2}(q \geq 1)$ of the progenitor binary.

In the next section we will estimate the amplitude of dominant modes by fitting the late time signal from a numerical relativity simulation to a superposition of ringdown modes. For binaries with non-spinning black holes considered in this paper we find that modes with $(\ell, m)=(2,2),(2,1),(3,3)$ and $(4,4)$ are the ones that are excited with amplitudes large enough to be interesting. For these modes, the angular functions $Y_{\ell m}^{+, \times}(\iota)$ are 
given by

$$
\begin{gathered}
Y_{+}^{22}(\iota)=\sqrt{\frac{5}{4 \pi}} \frac{\left(1+\cos ^{2} \iota\right)}{2}, \\
Y_{\times}^{22}(\iota)=\sqrt{\frac{5}{4 \pi}} \cos \iota, \\
Y_{+}^{21}(\iota)=\sqrt{\frac{5}{4 \pi}} \sin \iota, \\
Y_{\times}^{21}(\iota)=\sqrt{\frac{5}{4 \pi}} \cos \iota \sin \iota, \\
Y_{+}^{33}(\iota)=-\sqrt{\frac{21}{8 \pi}} \frac{\left(1+\cos ^{2} \iota\right)}{2} \sin \iota, \\
Y_{\times}^{33}(\iota)=-\sqrt{\frac{21}{8 \pi}} \cos \iota \sin \iota, \\
Y_{+}^{44}(\iota)=\sqrt{\frac{63}{16 \pi}} \frac{\left(1+\cos ^{2} \iota\right)}{2} \sin ^{2} \iota, \\
Y_{\times}^{44}(\iota)=\sqrt{\frac{63}{16 \pi}} \cos \iota \sin ^{2} \iota .
\end{gathered}
$$

In the case of binaries comprising of black holes with generic spins, the relative amplitudes of the various modes will also depend on the magnitude and direction of the spin vectors of the progenitor black holes. A detailed study of the dependence of the relative amplitudes of the various modes on the initial spin configurations and mass ratio of the progenitor binary is necessary to assess how accurately one might be able to use quasi-normal modes to measure a progenitor binary's parameters. For this, a more exhaustive set of simulations covering the full parameter space of binary black holes is required and will be taken up in the future.

We conclude this section by noting that using the expression for the two polarizations in Eq. (19) the detector response given in Eq. (8) can be written as:

$$
h^{A}(t)=\sum_{\ell, m>0} B_{\ell m} e^{-t / \tau_{\ell m}} \cos \left(\omega_{\ell m} t+\gamma_{\ell m}\right),
$$

where the superscript $A$ is an index denoting the detector in question (which is relevant when we have a network of detectors), $B_{\ell m}\left(\gamma_{\ell m}\right)$ is the following combination of the amplitudes $A_{\ell m}$ (respectively, phases $m \phi$ ), antenna pattern functions $F_{+}^{A}$ and $F_{\times}^{A}$ and the inclination angle $\iota$ :

$$
\begin{aligned}
& B_{\ell m}=\frac{\alpha_{\ell m} M}{D_{\mathrm{L}}} \sqrt{\left(F_{+}^{A} Y_{+}^{\ell m}\right)^{2}+\left(F_{\times}^{A} Y_{\times}^{\ell m}\right)^{2}}, \\
& \gamma_{\ell m}=\phi_{\ell m}+m \phi+\tan ^{-1}\left[\frac{F_{\times}^{A} Y_{\times}^{\ell m}}{F_{+}^{A} Y_{+}^{\ell m}}\right] .
\end{aligned}
$$

Note that, for the sake of clarity, we have dropped the index $A$ on $B_{\ell m}$ and $\gamma_{\ell m}$. Here, $\phi_{\ell m}$ are arbitrary con- stant phases of each quasi-normal mode 3 The effective amplitudes $B_{\ell m}$ are proportional to the intrinsic amplitudes $\alpha_{\ell m}$ of the modes and vary inversely with the luminosity distance. Their magnitude also depends on the various angles $(\theta, \varphi, \psi, \iota)$ describing the position of the source on the sky and its orientation relative to the detector through the antenna pattern functions $F_{+}$and $F_{\times}$ and spherical harmonic functions $Y_{+}^{\ell m}$ and $Y_{\times}^{\ell m}$. The constant phases $\gamma_{\ell m}$ also depend on the angles and the fiducial azimuth angle $\phi$.

The above form of the response is more helpful in understanding which, or which combination, of the parameters can be measured and how many detectors are required in solving the inverse problem, namely to fully reconstruct the incident gravitational wave and the parameters of the source that emitted the radiation. We shall use the above form of the waveform to compute the signal-to-noise ratios and the covariance matrix.

\section{AMPLITUDES OF MODES EXCITED DURING THE RINGDOWN PHASE OF A BLACK HOLE BINARY}

In this section we will use numerical simulations to evaluate the amplitude of the various modes excited as a function of the mass ratio $q$. We will examine how the amplitude of the dominant 22-mode varies as a function of $q$. Of particular interest would be the growth of 21-, 33 - and 44-modes relative to the 22 -mode as the binary system becomes more asymmetric. We will provide simple analytical fits to the amplitudes of all the different modes and discuss how the inclination angle of the black hole's final spin will affect the amplitude of the ringdown signal. Our analysis is complementary to previous studies of the mode structure of unequal-mass nonspinning binaries, for example [40, 53].

\section{A. Evolution of the luminosity}

An important question that arises in the study of QNMs excited during the merger of a black hole binary is the determination of the most dominant modes in the infinite mode sum in Eq. (1) for this particular kind of perturber. This analysis is necessary to construct a good model of the waveform to use in the analysis.

Figure 1 plots the luminosity in gravitational waves, $L_{\ell m}=D_{\mathrm{L}}^{2}\left[\left(\dot{h}_{+}^{\ell m}\right)^{2}+\left(\dot{h}_{\times}^{\ell m}\right)^{2}\right]$, in the first four most dominant modes as a function of the dimensionless time $t / M$, where $M$ is the initial total mass of the binary. The luminosities are plotted for four values of the mass ratio

\footnotetext{
${ }^{3}$ Specifically, the $\ell=m$ modes have a nearly consistent rotational phasing, while the $\ell \neq m$ modes seem to have somewhat distinct associated dynamics, with differentiated amplitude and phasing relationships during the merger process. 52
} 



FIG. 1. This plot shows the relative luminosities, or radiated power, in modes $(2,2),(3,3),(4,4)$, and $(2,1)$. $L_{l m}$ represent the luminosities (in units $c=G=1$ in which luminosity is dimensionless) and $r_{l m}$ denote the ratios $r_{l m}=L_{l m} / L_{22}$. The different panels correspond to systems with different mass ratios as indicated in the panel. Note that as the mass ratio increases, the luminosity in each mode decreases but the amplitudes of all higher-order modes relative to the $(2,2)$-mode increase. We have omitted - both in the figure and in this work — the next most dominant modes, $(5,5),(3,2),(4,3),(6,6)$ and $(5,4)$ as they are generally less than one percent as luminous as the $(2,2)$ mode. (see, however, Pan et al [51]).

$q=2,3,4$ and 11 . We have left out the plot corresponding to the equal mass case $q=1$, as in this case the modes with odd values of $l$ or $m$ are not excited and hence not as interesting as when the masses are unequal. The luminosity peaks just before the two black holes collide but different modes peak at different times. The 21- and 33 -modes peak after the 22-mode reaches its maximum. However, the 44-mode shows the opposite behaviour. For a more thorough investigation on the different multipolar contributions to the total radiated energy, see Ref. [52].

Although the 33-mode is absent when $q=1$, it is already more dominant than the 44-mode when $q=2$ and remains the most dominant after the 22-mode, throughout the inspiral and merger phase and for all mass ratios (except, of course, when $q=1$ ). The 44 -mode remains more dominant than the 21-mode for the most part, but the trend reverses after merger. This is because the 21mode reaches its peak luminosity a little after the 44mode. For $q=2$, when the 22 -mode reaches its peak, the luminosity in the 33-mode is an order-of-magnitude smaller than the dominant 22-mode; luminosities in 21and 44-modes are 50 times smaller than the 22-mode.

In addition to the luminosities, we have also plotted their ratios $r_{\ell m}=L_{\ell m} / L_{22}$ with respect to the 22-mode. It is clear that for more symmetric systems (i.e. $q \gtrsim 1$ ) higher modes are hardly excited. For instance, when $q=2$ the luminosity of the 21- and 44-modes remains below $\sim$ few percent of the 22 -mode, while the 33-mode is always less than $\sim 5 \%$ of the 22 -mode. As the mass ratio increases the higher order modes are excited with greater amplitudes. The different mode amplitudes become comparable to the 22-mode and to one another as the mass ratio increases. In the next two sub-sections we will give a more quantitative evaluation of the relative mode amplitudes in the ringdown part of the signal. 

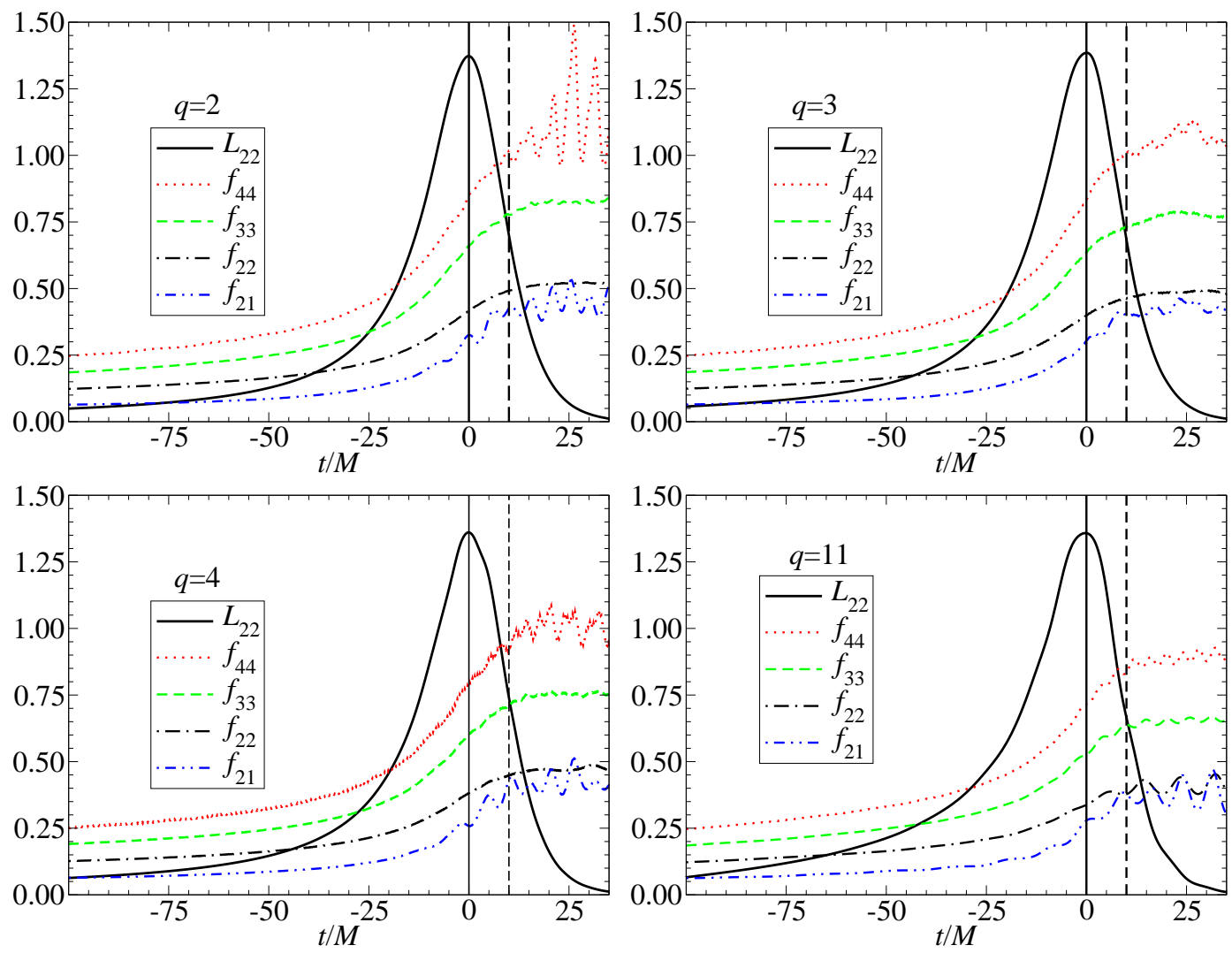

FIG. 2. Evolution of the first few dimensionless mode frequencies $f_{\ell m}=M \omega_{\ell m}$ as a function of the dimensionless time $t / M$, for different values of the mass ratio $q$ of the progenitor binary. Also shown in arbitrary units is the luminosity in the 22 mode. All mode frequencies, especially $f_{22}$ and $f_{33}$, stop evolving and stabilise soon after the binary merges to form a single black hole. The waveform is assumed to contain a superposition of only quasi-normal modes a duration $10 M$ after the luminosity in 22 mode reaches its peak.

\section{B. Identifying the ringdown phase}

An important task of our study is the identification of the point when the signal is purely a superposition of various quasi-normal modes. This is necessary in order that the proposed tests of general relativity are not corrupted due to the presence of extraneous signals. By assuming that the ringdown phase occurs sooner than it actually does, we are in danger of corrupting the waveform. Equally, by identifying the ringdown phase to be much later than it actually is, we will significantly weaken the tests since the signal amplitude falls off exponentially from the beginning of the ringdown phase. A proper identification of the beginning of the ringdown phase is needed to correctly extract the amplitude of the quasinormal modes and to compute the signal-to-noise ratio and other quantities.

To this end, we shall use the evolution of the frequency of the various modes $h_{\ell m}$. As a binary evolves, the frequency of each mode $h_{\ell m}$ increases, the rate of increase itself being greater as the two black holes get closer. When the two black holes merge, a common horizon forms and the frequency of each mode stabilizes, finally reaching the quasi-normal mode value as predicted by black hole per- turbation theory. We shall identify the beginning of the ringdown phase to be (approximately) the epoch when the frequency of the various modes begin to stabilize.

We can compute the frequency of each mode from the evolution of its phase given by the second of the equations in Eq. (14). Once the phase is known it is straightforward to write down the (dimensionless) frequency $f_{\ell m}=M \omega_{\ell m}=\mathrm{d} \Phi_{\ell m}(t) / \mathrm{d}(t / M)$. The ringdown phase can be assumed to begin when $f_{\ell m}$ computed from our numerical simulations are close to those obtained from black hole perturbation theory. We will first take a look at the predictions from black hole perturbation theory and then compare those predictions to the results obtained from our numerical simulations and plotted in Fig.2.

There has been a lot of work on the computation of the frequencies and time-constants of various modes of a perturbed Kerr black hole. Berti et al [18, have found simple fits, as a function of the spin parameter $j$, to the dimensionless mode frequencies $4 f_{\ell m}=M \omega_{\ell m}$ and qual-

\footnotetext{
${ }^{4}$ Note that we only consider here, the least damped $n=0$ overtone
} 
TABLE I. Dimensionless frequencies $f_{\ell m}$ for various modes for different values of the black hole spin $j$ that results from the merger of a binary of mass ratio $q$. We assume that the ringdown phase begins when the frequencies of various modes stop increasing and stabilize to a constant value. For each mode frequency, the column labelled "Fit" gives the values computed using Eqs. 27)-(30) and that labelled "NR" shows values at the "beginning" of the ringdown mode, a duration $10 M$ after the gravitational wave luminosity in the 22 mode reaches its peak (dashed vertical line in Fig.2).

\begin{tabular}{c|c|cc||cc||cc||cc||cc}
\hline \hline$q$ & \multirow{2}{*}{$j$} & \multicolumn{2}{|c||}{$f_{22}$} & \multicolumn{2}{c||}{$f_{21}$} & \multicolumn{2}{|c||}{$f_{33}$} & \multicolumn{2}{c}{$f_{44}$} \\
& & Fit & NR & Fit & NR & Fit & NR & Fit & NR \\
\hline 1 & 0.69 & 0.53 & 0.51 & 0.46 & - & 0.84 & - & 1.14 & 1.08 \\
\hline 2 & 0.62 & 0.50 & 0.49 & 0.44 & 0.42 & 0.80 & 0.78 & 1.09 & 1.05 \\
\hline 3 & 0.54 & 0.48 & 0.47 & 0.43 & 0.41 & 0.76 & 0.74 & 1.03 & 1.01 \\
\hline 4 & 0.47 & 0.46 & 0.45 & 0.42 & 0.43 & 0.73 & 0.72 & 0.99 & 0.97 \\
\hline 11 & 0.25 & 0.41 & 0.39 & 0.39 & 0.41 & 0.66 & 0.64 & 0.89 & 0.85 \\
\hline \hline
\end{tabular}

ity factors $2 Q_{\ell m}=\tau_{\ell m} \omega_{\ell m}$. The fitting functions for the $22,21,33$ and 44 modes are given by [18]

$$
\begin{aligned}
f_{22} & =1.5251-1.1568(1-j)^{0.1292} \\
Q_{22} & =0.7000+1.4187(1-j)^{-0.4990} \\
f_{21} & =0.6000-0.2339(1-j)^{0.4175} \\
Q_{21} & =-0.3000+2.3561(1-j)^{-0.2277}, \\
f_{33} & =1.8956-1.3043(1-j)^{0.1818} \\
Q_{33} & =0.9000+2.3430(1-j)^{-0.4810} \\
f_{44} & =2.3000-1.5056(1-j)^{0.2244} \\
Q_{44} & =1.1929+3.1191(1-j)^{-0.4825} .
\end{aligned}
$$

These fits are quite robust and they differ from the actual values obtained for the frequencies and quality factors by no more than $3 \%$ [18. Table $\mathrm{I}$ lists frequencies $f_{22}, f_{21}$, $f_{33}$ and $f_{44}$, for several different values of the spin parameter $j$. The chosen values of $j$ correspond to the final spins of black holes that result in our numerical simulations of binaries with different mass ratios $q$. Values in columns labelled "Fit" are those obtained using Eqs. (27)-(30) and those in columns labelled "NR" are those obtained from our numerical relativity simulations as follows.

Figure 2 plots the frequencies $f_{\ell m}$ for the 22, 21, 33, and 44 modes as (black) dot-dashed, (blue) dot-dotdashed, (green) dashed and (red) dotted curves. As expected, the frequency of each mode increases, quite rapidly towards the end, but stabilizes to a constant value - the quasi-normal mode frequency of the final black hole. The plots also show the (arbitrarily scaled) luminosity in

for each mode and have therefore dropped the overtone index from mode frequencies, quality factors and time-constants. the 22-mode as a (black) solid curve. The epoch at which the luminosity reaches its peak (indicated by a solid vertical line) has been set to be $t / M=0$. We see that the onset of the ringdown phase occurs significantly after the luminosity reaches its peak. For simplicity, we have chosen the beginning of the ringdown phase to be a duration $t=10 M$ after the system reaches its peak luminosity, indicated by a dashed vertical line in Fig. 2. In reality for the $\ell=m$ modes, it is $4 M-5 M$ earlier than for the $(2,1)$ mode. The luminosity curves, Fig.1 1 exhibit a similar behaviour. See also [52].

Most modes seem to stabilize at the onset of the ringdown phase. For $q \lesssim 4$ the frequency of 22 and 33 modes stabilize, but it is less so with 21 and 44 modes. In our $q=11$ simulations all mode frequencies seem to oscillate around a mean value, 22 and 21 more than 33 and 44 . From Fig.11 we see that after reaching the peak luminosity the amplitudes of 21 and 33 modes relative to the 22 mode (thick lines) are constant. This justifies why we might fit a $q$-dependent function to the relative amplitudes of various modes (see Sec. IV Cland Fig. 3) that is valid throughout the ringdown phase. The same cannot be said about the 44 mode.

Under columns labelled "NR", Table 1 gives frequencies $f_{22}, f_{21}, f_{33}$ and $f_{44}$ at the onset of the ringdown mode (i.e., an epoch $t=10 M$ after the luminosity of the 22 mode reaches its peak) obtained from our numerical relativity simulations. Modes with odd $\ell$ or $m$ are not excited when a binary comprising a pair of equal mass black holes merges, which is the reason why these entries are missing from the Table. The mode frequencies at $10 M$ after peak luminosity agree with the fits obtained from black hole perturbation theory to within $5 \%$. Hence we believe that our method is quite robust in identifying the ringdown phase.

\section{Relative amplitudes in the ringdown phase}

Our goal here is to estimate the amplitudes of the various modes in the ringdown phase. The transition from the inspiral to the ringdown phase is very smooth and it is not easy to pick a unique instant after which the transition occurs. Amplitudes of the various modes in the ringdown phase are given in Table II at three different epochs:

- at the epoch when the luminosity of the 22 mode reaches its peak,

- a duration $10 M$ after the 22 mode reaches its peak luminosity, and

- a duration $15 M$ after the 22 mode reaches its peak luminosity.

The Table lists the absolute amplitude $\alpha_{22}$ of the 22 mode and relative amplitudes $\alpha_{\ell m} / \alpha_{22}$ of the rest of the modes. These amplitudes are plotted in Fig. 3 . 
TABLE II. For different mass ratios (column 1), we list the final spin $j$ of the black hole (column 2), amplitudes of different modes at three different epochs: (i) when the 22 mode reaches its peak luminosity (columns 3-6), an epoch 10M (columns 7-10) and $15 \mathrm{M}$ after the 22 mode reaches its peak luminosity. We list the absolute value of the amplitude for the 22 mode and the ratio of amplitudes of the rest of the modes to the 22 mode. The beginning of ringdown, taken as the point when the instantaneous frequency of each mode begins to stabilize, is typically found to be $\sim 1-2$ cycles after the peak luminosity. For concreteness we take the beginning of the ringdown mode to be $10 M$ after the peak luminosity.

\begin{tabular}{|c|c|c|c|c|c|c|c|c|c|c|c|c|c|}
\hline \multirow[t]{2}{*}{$q$} & \multirow[t]{2}{*}{$j$} & \multicolumn{4}{|c|}{ At peak luminosity } & \multicolumn{4}{|c|}{ At $10 M$ after peak } & \multicolumn{4}{|c|}{ At $15 M$ after peak } \\
\hline & & $\alpha_{22}$ & $\alpha_{33} / \alpha_{22}$ & $\alpha_{44} / \alpha_{22}$ & $\alpha_{21} / \alpha_{22}$ & $\alpha_{22}$ & $\alpha_{33} / \alpha_{22}$ & $\alpha_{44} / \alpha_{22}$ & $\alpha_{21} / \alpha_{22}$ & $\alpha_{22}$ & $\alpha_{33} / \alpha_{22}$ & $\alpha_{44} / \alpha_{22}$ & $\alpha_{21} / \alpha_{22}$ \\
\hline 1 & 0.69 & 0.365 & 0.000 & 0.052 & 0.000 & 0.217 & 0.000 & 0.043 & 0.000 & 0.152 & 0.000 & 0.038 & 0.000 \\
\hline 2 & 0.62 & 0.321 & 0.149 & 0.050 & 0.114 & 0.194 & 0.161 & 0.030 & 0.121 & 0.132 & 0.156 & 0.020 & 0.135 \\
\hline 3 & 0.54 & 0.266 & 0.216 & 0.070 & 0.178 & 0.158 & 0.247 & 0.052 & 0.195 & 0.108 & 0.244 & 0.044 & 0.212 \\
\hline 4 & 0.47 & 0.225 & 0.259 & 0.087 & 0.203 & 0.140 & 0.267 & 0.069 & 0.234 & 0.095 & 0.264 & 0.057 & 0.262 \\
\hline 11 & 0.25 & 0.100 & 0.349 & 0.156 & 0.312 & 0.063 & 0.377 & 0.154 & 0.407 & 0.048 & 0.347 & 0.137 & 0.478 \\
\hline
\end{tabular}

First let us note that although, as expected, the absolute amplitude of the 22 mode depends on the epoch that we identify as the start of the ringdown phase, the amplitudes of the sub-dominant modes relative to the 22 mode are not too sensitive to that identification. This is especially true for the 33 and 44 modes whose peak luminosity is at the same epoch as that of the 22 mode [thin (green) dashed and (red) dotted curves in Fig. 1], but less so for the 21 mode thick (blue) dot-dot-dashed curve in Fig. 1. bottom right panel of Fig. 3, whose peak luminosity occurs significantly after that of the 22 mode. As mentioned before, for concreteness we shall take the starting point of the ringdown mode as an epoch $10 \mathrm{M}$ after the 22 mode reaches its peak luminosity (the dashed vertical line in Fig. 2). All discussions in the remainder of this paper are based on this identification. A duration of $10 M$ corresponds to between 1 and 2 gravitational-wave cycles of the merger signal.

As the mass ratio of the progenitor binary increases, the amplitude of the 22 mode rapidly decreases but the sub-dominant modes approach each other in power (see Fig. 1) and their amplitudes increase (cf. Table II and Fig. 3). For a mass ratio of $q=4$, the 33 and 21 modes have amplitudes $1 / 4$ that of 22 while at $q=11$ they are $40 \%$ of the 22 mode. Of course, the overall luminosity decreases as the mass ratio increases and one expects no radiation in the limit $q \rightarrow \infty$. Indeed, the emitted energy during the 'merger' phase goes roughly as the square of the symmetric mass ratio $\nu=m_{1} m_{2} / M^{2}$ of the progenitor binary [40].

Let us note that the values in Table $\amalg$ do not all refer to the same final black hole spin. All our black holes are initially non-spinning and the final spin is simply the residual angular momentum of the progenitor binary. The final spin, therefore, depends on the mass ratio, and is greatest when the two black holes are of the same mass. In principle, it should be possible, but in practice very difficult, to produce numerical data for different mass ratios all with the same final spin. To do so we require an accurate mapping between the mass ratio and initial black-hole spins, and the spin of the final black hole.
For example, configurations that lead to a nonspinning Schwarzschild black hole are suggested in Ref. [54 and more generic cases are considered in Refs. [55] 58]. However, fine-tuning the spin of the final black hole requires that the component black holes are also spinning, and in this paper we consider only binaries with non-spinning components.

\section{Fitting functions for relative amplitudes}

For the purpose of computing the signal-to-noise ratio and the covariance matrix it is convenient to have analytical expressions for the relative amplitudes of the various modes. Our fits are meant to capture the dependence of the amplitudes on the mass ratio in the range we have considered in this paper. They are not meant to explore the complex dynamics of a ringing black hole and are not necessarily valid outside the region we have explored.

We have seen that the amplitudes inferred from the simulations depend on how we identify the beginning of the ringdown phase. As mentioned before, in our calculations we have assumed that the ringdown phase begins an epoch $10 M$ after the 22 mode reaches its peak luminosity. We find that the amplitude of the 22 mode at this epoch as a function of the mass ratio is well fitted by

$$
\alpha_{22}(q)=0.25 e^{-q / 7.5} .
$$

The solid curve in the top left panel in Fig. 3 shows that this is a good fit to the data points (filled squares). Dotdot-dashed and dotted lines in the same panel show the fits that describe $\alpha_{22}(q)$ at the peak of the luminosity and $15 M$ after the peak, respectively. In all cases, the data is pretty well approximated by an exponentially falling function.

The relative amplitudes of the sub-dominant 21, 33 and 44 modes are well fitted by the following functions

$$
\begin{aligned}
& \alpha_{21}(q)=0.13 \alpha_{22}(q)(q-1)^{1 / 2}, \\
& \alpha_{33}(q)=0.18 \alpha_{22}(q)(q-1)^{1 / 3}, \\
& \alpha_{44}(q)=0.024 \alpha_{22}(q) q^{3 / 4} .
\end{aligned}
$$






FIG. 3. This plot shows the amplitudes as a function of the mass ratio for different modes at the peak of the luminosity of the 22 mode (circles), an epoch $10 M$ and $15 M$ after the peak (respectively, squares and triangles). We have plotted the absolute amplitude $\alpha_{22}$ of the 22 mode and ratio of the subdominant mode amplitudes $\alpha_{\ell m} / \alpha_{22}$ relative to 22 (cf. Table II). The solid lines are the best fits [cf. Eqs. (31)-(34)] to the amplitudes at $10 M$ after the peak luminosity.

They are plotted as solid curves in the relevant panels of Fig. 3. The fits are motivated by the fact that when $q=1$ only modes with even values of $\ell$ and $m$ are excited, while those with odd $\ell$ or $m$ are absent. Note that the relative amplitudes grow as a binary becomes more asymmetric and so higher order modes should be more easily detectable if the mass ratio is large. In absolute terms, of course, all modes are exponentially damped as a function of $q$.

In this work we estimated the relative amplitudes of different modes at a single epoch. It might be more reliable to estimate their average over a small duration, for instance between $10 M-15 M$. This could diminish any 'numerical noise' present and extract more accurate fits but we did not explore this alternative approach in this work.

\section{VISIBILITY OF RINGDOWN MODES}

In this section we will study the signal-to-noise ratio (SNR) obtained by various detectors for the ringdown phase of the coalescence of a black hole binary. We will begin by defining the matched filter SNR, followed by the noise power spectral densities of aLIGO, ET and LISA and the choice of signal parameters used in the study. We will then discuss the visibility of the different modes, focusing on the distance reach of the various detectors.

\section{A. Matched filter SNR}

The matched filter SNR $\rho$ obtained while searching for a signal of known shape buried in Gaussian background is given by (see, e.g., [59])

$$
\rho^{2}=4 \int_{0}^{\infty} \frac{|H(f)|^{2}}{S_{h}(f)} \mathrm{d} f
$$

where $S_{h}(f)$ is the detector noise power spectral density and $H(f)$ is the Fourier transform of the signal assumed (in this work) to be a superposition of the 22, 21 and 33 modes. In the time-domain our waveform is given by Eq. 244 where the sum is over $(\ell, m)=(2,2),(2,1),(3,3)$ and we have ignored all higher order modes including the $(4,4)$ mode. The coefficients $\alpha_{\ell m}$ required to compute the waveform are assumed to be as in Eqs. (31)-(33).

The ringdown signal is a superposition of different modes and its visibility depends not only on the relative amplitudes but also on the relative phases of the different modes. To this end, it is useful to define the SNR of a mode as

$$
\rho_{\ell m}^{2}=4 \int_{0}^{\infty} \frac{\left|H_{\ell m}(f)\right|^{2}}{S_{h}(f)} \mathrm{d} f
$$

with the caveat that the total SNR $\rho^{2}$ is not the quadrature sum of $\rho_{\ell m}^{2}$ since there are also interference terms that can be negative.

\section{B. Sensitivity curves}

In our study, we will consider the performance of three detectors: the aLIGO ET and LISA. A fit to the aLIGO noise spectral density tuned to detect binary neutron stars is 5

$$
\begin{aligned}
S_{h}(f) & =10^{-49}\left[10^{16-4(f-7.9)^{2}}+0.08 x^{-4.69}\right. \\
& \left.+123.35 \frac{1-0.23 x^{2}+0.0764 x^{4}}{1+0.17 x^{2}}\right] \mathrm{Hz}^{-1}
\end{aligned}
$$

where $x=f / 215 \mathrm{~Hz}$. In the case of ET we consider the sensitivity curve designated ET-B [60] whose noise power spectral density is given by $S_{h}(f)=10^{-50} h_{n}(f)^{2} \mathrm{~Hz}^{-1}$

$$
\begin{aligned}
h_{n}(f) & =2.39 \times 10^{-27} x^{-15.64}+0.349 x^{-2.145} \\
& +1.76 x^{-0.12}+0.409 x^{1.10},
\end{aligned}
$$

where $x=f / 100 \mathrm{~Hz}$. We take LISA noise spectral density to be the one that was used by the LISA Parameter Estimation Taskforce in Ref. [61, which also corresponds to the noise curve from the second round of the Mock LISA Data Challenge [62, 63].

\footnotetext{
${ }^{5}$ The fit was provided by C. Capano, Syracuse University.
} 



FIG. 4. The signal-to-noise ratio integrand for LISA for a quasi-normal mode signal that is composed of 22,21 and 33 modes - the three most dominant ones. The source is assumed to be at a red-shift of $z=1$ and the various angles are as in Table III The left panel corresponds to a black hole of mass $M=5 \times 10^{6} M_{\odot}$ and the right panel to a black hole of mass $M=10^{7} M_{\odot}$. In both cases the mass ratio of the progenitor binary is taken to be $q=10$.

\section{Choice of various parameters}

The SNR depends on a number of source parameters as well as the location of the source on the sky. We have limited our investigations to studying the SNR and covariance matrix as a function of the black hole's (observed) mass $M$ and the mass ratio $q$ of the progenitor binary, for fixed values of the distance to the black hole and various angles. In the case of LISA the black hole is assumed to be at a red-shift of $z=1$ which corresponds (in our cosmological model) to a luminosity distance of $D_{\mathrm{L}} \simeq 6.73 \mathrm{Gpc}$. In the case of aLIGO and ET we set $D_{\mathrm{L}}=1 \mathrm{Gpc}$. In all cases the angles are fixed to be $\theta=\psi=\iota=\varphi=\pi / 3$. For a statistical analysis of the effect of these angular parameters on the detectability of a ringdown signal see 64 .

The black hole mass is varied over the range $\left[100,10^{3}\right] M_{\odot}$ in the case of aLIGO, $\left[10,10^{3}\right] M_{\odot}$ in the case of ET and $\left[3 \times 10^{6}, 10^{8}\right] M_{\odot}$ in the case of LISA. These choices are dictated by the frequency sensitivity of the instruments which further dictates the range of black hole masses whose ringdown radiation they are most sensitive to.

Our choice of parameters is summarized in Table III. We reiterate that our masses are observed masses which means that the intrinsic mass of the black hole is smaller by a factor $1+z \simeq 1.2$, for aLIGO and ET and by a factor of $1+z=2$ in the case of LISA. Although the signal visibility simply scales as the inverse of the distance, the fact that the mass is blue-shifted means that we cannot easily scale our results to another (say, a greater) distance for the same intrinsic masses. Such a scaling will be valid if at the same time the intrinsic masses are also scaled up/down by the appropriate red-shift factor.
TABLE III. This Table lists the values of the various parameters used in our study. In all cases the angles are all set to $\theta=\varphi=\psi=\iota=\phi=\pi / 3$.

\begin{tabular}{cccc}
\hline \hline Detector & $D_{\mathrm{L}} / \mathrm{Gpc}$ & $M / M_{\odot}$ & $q$ \\
\hline aLIGO & 1.00 & {$\left[100,10^{3}\right]$} & $2-10$ \\
\hline ET & 1.00 & {$\left[10,10^{3}\right]$} & $2-10$ \\
\hline LISA & 6.73 & {$\left[3 \times 10^{6}, 10^{8}\right]$} & $2-25$ \\
\hline \hline
\end{tabular}

\section{Visibility of different modes}

It is instructive to plot the SNR integrand $\mathrm{d} \rho^{2} / \mathrm{d} f=$ $|H(f)|^{2} / S_{h}(f)$ as it depicts how the different modes become important for systems with different masses. Fig.4 4 plots this quantity for two systems as seen in LISA. The left panel corresponds to a black hole of total mass $M=5 \times 10^{6} M_{\odot}$ and the right panel to $M=10^{7} M_{\odot}$. The mass ratio $q$ is $q=10$ in both cases and the angles are as in Table 【II.

The various mode frequencies $F_{\ell m}=\omega_{\ell m} /(2 \pi)$ of the two systems are $F_{22} \simeq 2.74 \mathrm{mHz}, F_{21} \simeq 2.54 \mathrm{mHz}$ and $F_{33} \simeq 4.26 \mathrm{mHz}$ for the lighter black hole and $F_{22} \simeq$ $1.37 \mathrm{mHz}, F_{21} \simeq 1.27 \mathrm{mHz}$ and $F_{33} \simeq 2.13 \mathrm{mHz}$ for the heavier black hole. Let us first note that the 22 mode of the lighter black hole and 33 mode of the heavier black hole are close to the region where LISA has best sensitivity. This will be relevant in the discussion that follows.

The intrinsic amplitudes of the 21 and 33 modes are a little more than a third of the 22 mode for $q=10$. However, since the SNR integrand depends on the signal power weighted down by the noise power, for a given black hole mass the SNR integrand could be as large as, or even dominated by, modes different from the 22 mode. This does not happen for the 21 mode since the frequencies of the 22 and 21 modes are very close to each other and so the 21 mode is always far smaller than the 


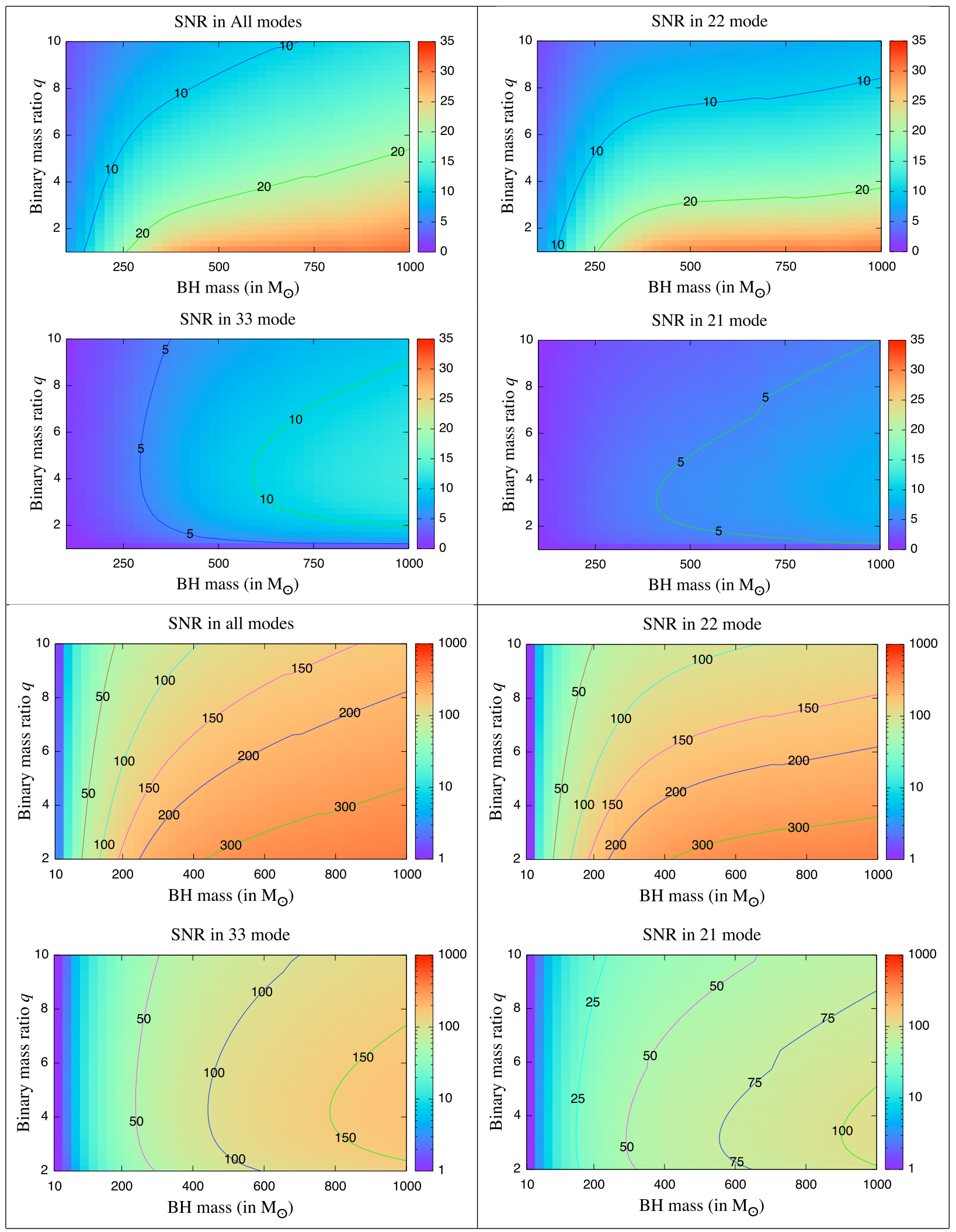

FIG. 5. Signal-to-noise ratio (SNR) in Advanced LIGO (top set of four panels) and Einstein Telescope (bottom set of four panels) as a function of the black hole's mass $M$ and progenitor binary's mass ratio $q$ for different modes. Most of the contribution to the SNR comes from the 22 mode but other modes too have significant contributions, 33 being more important than 21. The source is assumed to be at a distance of $1 \mathrm{Gpc}$ and various angles are as in Table III. 
22 mode. For a black hole mass of $10^{7} M_{\odot}$ the 33 mode is as strong as the 22 mode and for masses even larger, the 33 mode overwhelms the 22 mode. The total SNR for the $5 \times 10^{6} M_{\odot}$ black hole is $\rho=1670$, with the different modes contributing $\rho_{22}=1500, \rho_{21}=625$, and $\rho_{33}=$ 950. The SNR is clearly dominated by the 22 mode.

In the case of the heavier black hole, the total SNR is $\rho=2520$, with the different modes contributing $\rho_{22}=$ $1940, \rho_{21}=920, \rho_{33}=1860$. In this case, the 33 mode is as strong as the 22 mode but the 21 mode, as expected, is sub-dominant.

\section{E. Exploring black hole demographics with ET and LISA}

Figures 5 and 6 plot the SNR in the ringdown signal (plots titled "SNR in all modes") and contribution from the 22, 21 and 33 modes (plots titled accordingly) as a function of the black hole mass $M$ and mass ratio $q$ of the progenitor binary for aLIGO, ET and LISA; $M$ and $q$ are varied over the range as in Table III] Most of the contribution to the SNR comes from the 22 mode followed by 33 and 21. Let us recall that SNRs from different modes do not add in quadrature.

In the case of aLIGO, the 22 and 33 modes will be visible in a significant fraction of the parameter space explored provided the source is within a distance of $1 \mathrm{Gpc}$. The 21 mode will not be visible in aLIGO at this distance except perhaps for the heaviest systems explored.

In the case of ET, assuming a SNR threshold of 10 for detection, the signal is visible to a red-shift of $z \sim 0.8$ in most of the parameter space explored. Black holes of total mass $M>400 M_{\odot}$ that form from the coalescence of binaries whose mass ratio is less than 4 will be visible at red-shifts $z \sim 2-3.5$

In the case of LISA, ringdowns produce a very large SNR. Even assuming an SNR threshold of 40, LISA should see the formation of supermassive black holes in the range $\left[10^{6}, 10^{8}\right] M_{\odot}$ up to a red-shift of at least $z \sim 6$ but if the progenitor black holes have mass ratio $q<10$ they should be visible from the earliest moments of their formation in the Universe.

Our results unambiguously demonstrate that ET and LISA can together probe black hole demographics, ET exploring the lower end of the mass spectrum of seed black holes and LISA the higher end of that spectrum. The two detectors together cover a large mass range from $\sim 10^{2} M_{\odot}$, all the way to $\sim 10^{8} M_{\odot}$. (Although out of the range of masses explored, note that ET could observe heavier black holes of mass $10^{4} M_{\odot}$ and LISA could explore lighter black holes of mass $10^{5} M_{\odot}$.) The distance reach will be different depending on the mass ratio of the progenitor binary and the total mass of the black hole. Even so, LISA and ET will make it possible to explore the formation of black holes and trace their merger histories and possibly help understand the role of black holes as seeds of galaxies and large scale structure in the
Universe.

\section{WHAT CAN A RINGDOWN SIGNAL MEASURE?}

By measuring the ringdown signal and resolving it into different modes, we should be able to learn a great deal about the merger dynamics and test general relativity. For instance, by determining the total mass of the binary from the inspiral phase and comparing it to the mass of the black hole obtained from the ringdown phase we can measure, quite precisely, how much mass is converted into radiation in the process of merger. LISA can typically measure the total mass of a binary from its inspiral phase to a fraction of a percent. We shall see in this section that the ringdown modes can determine a black hole's mass to a percent or tenth of a percent depending on the mass ratio. Therefore, the inspiral and ringdown phases together can shed light on how much mass is lost in the process of merger and how that depends on the mass ratio of the binary and, not probed in this study but expected to depend on, the spin magnitudes and orientations of progenitor black holes.

When black holes merge, some of the orbital angular momentum goes into the final black hole. Therefore, the final black hole will spin in a direction different from either of the progenitor black holes. Independent measurements of the orbital angular momentum from the inspiral phase and black hole spin from the ringdown phase, could unravel the spin-orbit dynamics of black hole merger. While this is an exciting possibility, in this paper we have focussed only on binaries with non-spinning components. For such systems, the relative amplitudes of the different modes depend on only the mass ratio of the progenitor binary. For binaries with spinning black holes it is hard to guess how many more parameters might be required to characterize the relative amplitudes and hence their measurability. We will address this question in a forthcoming publication. We shall show in this section that one can exploit this fact to determine the mass ratio of the progenitor binary from the measurement of the ringdown mode alone. Consistency of the mass ratios from the inspiral and ringdown phases could offer further tests of general relativity.

In this section we will explore what measurements might be possible by using ringdown signals alone and what we might learn by combining the information obtained from the inspiral phase of the signal with that obtained from the ringdown phase. To this end, we shall assume that the signals are loud and compute the covariance matrix to get an estimate of the measurement uncertainties in the various parameters of a ringdown signal $h^{A}(t)$. The covariance matrix $C_{k m}^{A}$ is the inverse of the Fisher matrix $F_{k m}^{A}$ given by 6567 ,

$$
F_{k m}^{A}=\left\langle\frac{\partial h^{A}}{\partial \lambda^{k}}, \frac{\partial h^{A}}{\partial \lambda^{m}}\right\rangle, \quad \lambda^{k}=\{M, j, q, \ldots\}
$$




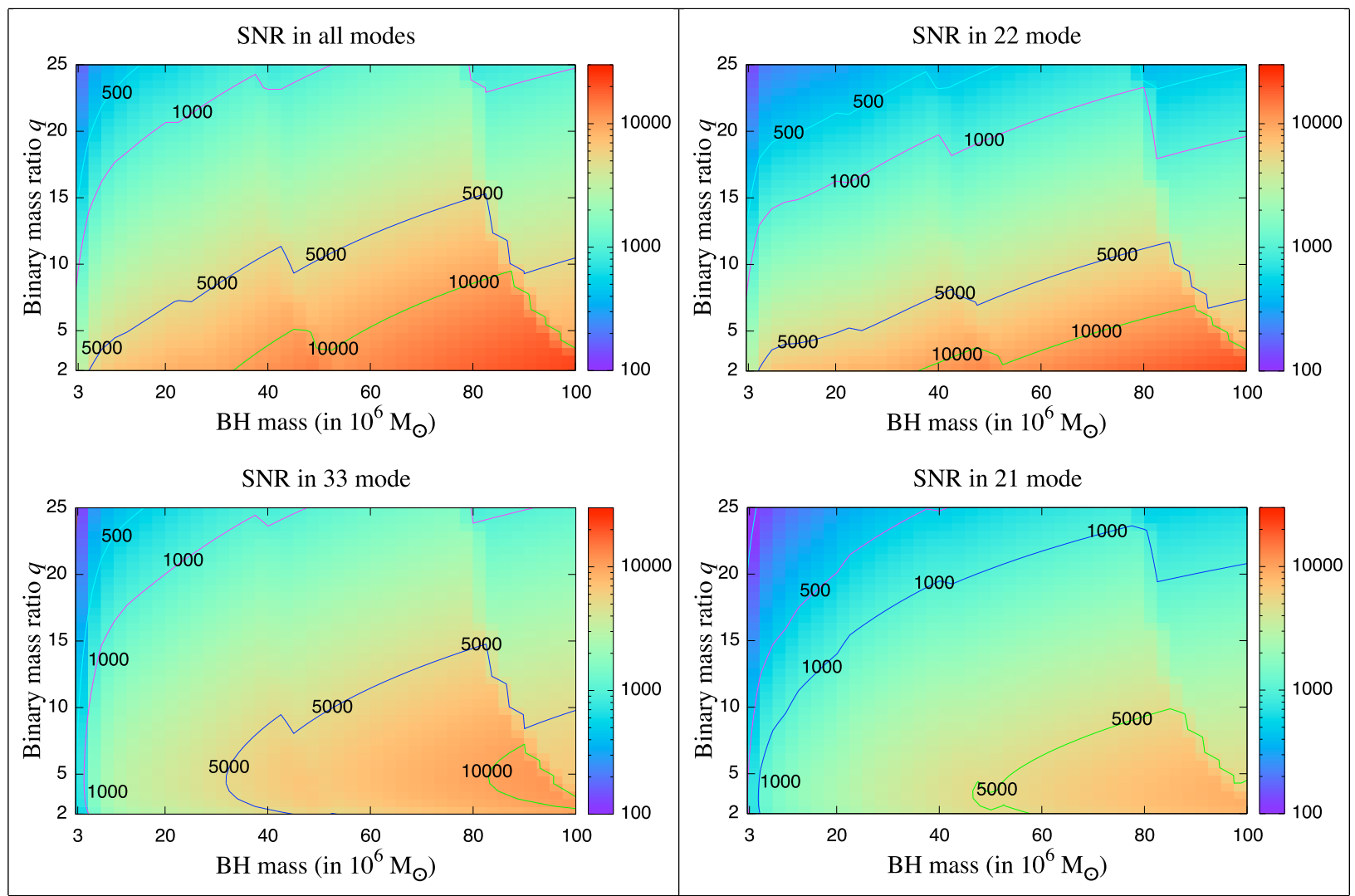

FIG. 6. Same as Fig. 5 but for LISA and the source is assumed to be at a red-shift of $z=1$. Also, the mass ratio is allowed to vary over a larger range from 2 to 25 instead of 2 to 10 . The 'steps' that can be seen around $40 \times 10^{6} M_{\odot}$ and $80 \times 10^{6} M_{\odot}$ are mostly due to the LISA noise curve.

where for any two functions $g(t)$ and $h(t)$ the angular bracket $\langle g, h\rangle$ denotes their scalar product defined by

$$
\langle g, h\rangle=4 \Re \int_{0}^{\infty} G(f) H^{*}(f) \frac{\mathrm{d} f}{S_{h}(f)} .
$$

Here, as before, $A$ is an index denoting the detector in question, $S_{h}(f)$ is the one-sided noise power spectral density of the detector, $G(f)$ and $H(f)$ are the Fourier transforms of the time-domain functions $g(t)$ and $h(t)$, respectively, and a $*$ denotes the complex conjugate of the quantity in question. The above integrals are often performed numerically and it is essential then to appropriately choose the lower and upper limits in the integral so that outside this limit the integral has negligible contribution. Note that the detector noise power spectral density rises steeply outside a certain frequency range often assuring the convergence of these integrals. If a network of detectors is used to estimate the parameters then the Fisher matrix for the network is simply the sum of the Fisher matrices for the individual detectors:

$$
F_{k m}=\sum_{A} F_{k m}^{A}
$$

where the sum is over all the detectors in the network.

\section{A. The full parameter set}

In the case of a binary consisting of non-spinning black holes on a quasi-circular orbit, the effective amplitudes $B_{\ell m}$ in Eq. (24) of the quasi-normal modes of the final black hole, depend on a set of eight parameter ${ }^{6}$ $\left(M, j, q, D_{\mathrm{L}}, \theta, \varphi, \psi, \iota\right):$ the mass $M$ and spin magnitude $j$ of the black hole, the mass ratio $q$ of the progenitor binary, the position vector $\left(D_{\mathrm{L}}, \theta, \varphi\right)$ of the black hole with respect to Earth, the polarization angle $\psi$ and the inclination $\iota$ of the black hole's spin angular momentum with respect to the line-of-sight. The phases $\gamma_{\ell m}$ are given by Eq. (26) and they depend on the angles $\left(\theta, \varphi, \psi, \iota, \phi, \phi_{\ell m}\right)$, where $(\iota, \phi)$ are the spherical polar coordinates giving the direction in which the black hole quasi-normal mode is emitted in a frame fixed to the black hole and $\phi_{\ell m}$ are the initial phases of different quasi-normal modes. Thus, if we consider a superposi-

\footnotetext{
${ }^{6}$ Recall that the final spin of the black hole is determined by the mass ratio of the progenitor binary and so it is not necessary to treat both $q$ and $j$ as independent. However, such a treatment allows us to check if the final black hole spin is consistent with the mass ratio as predicted by numerical relativity simulations, which would indeed be another test of general relativity.
} 
tion of three quasi-normal modes then there will be 12 parameters, including $\phi_{22}, \phi_{33}$ and $\phi_{21}$. The amplitudes $B_{\ell m}$ depend on eight of these parameters (exceptions are $\phi, \phi_{22}, \phi_{33}$ and $\left.\phi_{21}\right)$ and phases $\gamma_{\ell m}$ also depend on a (different) set of eight parameters (exceptions are $D_{\mathrm{L}}$, $M, q$, and $j$ ).

\section{B. Measurements with a network of detectors}

Measuring all the parameters of a ringdown signal will require simultaneous observation of the signal in two or more detectors. Let us first take a look at the configurations of LISA, ET and advanced ground-based detectors.

ET and LISA both have a triangular topology and consist of three V-shaped interferometers, with an opening angle of 60 degrees, rotated relative to each other by 120 degrees. The three interferometers are completely equivalent, in terms of sensitivity, to two L-shaped interferometers 68, with arms that are only three-quarters in length of the arms in the triangle. Thus, for the purpose of detection and measurement, we can consider ET and LISA to be a network of two collocated detectors. At least three advanced ground-based detectors (two LIGO detectors and Virgo) would be operating by 2015, with the possibility of the Japanese Large Cryogenic Gravitational Telescope joining the network soon after. Thus, there will be a global network of ground-based detectors that will be operating for a number of years from around 2015.

Of all the parameters, the direction to the source $(\theta, \varphi)$ is the most critical and difficult to measure from the ringdown modes alone. However, since the ringdown modes we study are preceded by the inspiral phase of a binary coalescence, we can expect the direction to the source to have been measured to some degree of accuracy. In the case of LISA, the inspiral phase of supermassive black holes could last for several months to years in the detector band. The modulation of the signal caused by LISA's motion relative to the source over the observation period will be good enough to measure the sky position (see, for instance, Ref. [69]). ET, together with a network of other detectors, advanced or 3rd generation detectors present at the time, should be able to triangulate the source. This is also true with the network of advanced detectors. Thus, we shall assume that the parameters $(\theta, \varphi)$ are known, leaving 10 parameters to be measured from the ringdown phase. However, for very massive systems (depending on the detector in question), only the ringdown phase might be visible and for such systems it will not be possible to infer the location of the source without a network of detectors. Such events will not be very useful for testing GR.

As expected, the relative contributions of the inspiral and ringdown phases depend on the total mass of the system: For lighter masses the ringdown phase makes little impact on parameter estimation; for heavier systems just the opposite is true. For systems with intermediate masses the contributions could be roughly equal. Such systems will be golden binaries with the best ability to test GR.

A single detector can measure the mass $M$ and spin $j$ of the black hole by simply inverting the QNM frequencies and damping times. Additionally, each detector in a network would also measure three independent amplitudes $B_{22}^{A}, B_{21}^{A}$ and $B_{33}^{A}$ and three independent phases $\gamma_{22}^{A}, \gamma_{21}^{A}$ and $\gamma_{33}^{A}$ - a set of 12 additional measurements from two detectors. Of course, the amplitudes and phases (as well as the time-constants and mode frequencies) are all expressed in terms of the 10 physical parameters and will not treated as independent. The counting argument given here shows that a set of two or more detectors allows enough measurements to fully reconstruct the ringdown signal.

Therefore, one can, in principle, measure all the ten parameters of a QNM composed of three modes, using a network of two or more detectors. We have not, however, explored the problem in its full generality as the Fisher matrix that includes both intrinsic and extrinsic parameters happens to be highly ill-conditioned. In such cases, fisher matrix is not the right approach for computing the errors incurred. We will, in the near future, investigate this problem by other means, for instance using Bayesian inference. For now, our goal is to see how well a subset of interesting parameters can be measured if, as mentioned earlier, we know some of the parameters from the inspiral phase.

For the sake of simplicity, we shall assume that the phase of the different quasi-normal modes at the beginning of the ringdown are all the same and equal to zero: $\phi_{22}=\phi_{21}=\phi_{33}=0$. In this work, we have dropped them from further consideration so that we can focus our effort on the main goal of the paper, which is to show that one can infer the mass ratio of the progenitor binary by measuring two or more quasi-normal mode amplitudes. We shall, therefore, assume that the ringdown signal depends on the parameters $\left(M, j, q, D_{\mathrm{L}}, \iota, \psi, \phi\right)$, seven parameters in all. A single detector can measure the mass and spin of the black hole from the different mode frequencies and damping times, as well as three amplitudes and three phases. Consequently, in the case of a simplified signal model, where we have dropped the constant phases and the location of the black hole from the list of parameters, we do not need a network of detectors to resolve the signal parameters.

\section{UNDERSTANDING MASS LOSS, SPIN RE-ORIENTATION AND MASS RATIO FROM RINGDOWN SIGNALS}

We have computed the covariance matrix $C_{k m}$ [cf. Eq. [39p] of ringdown signals as a function of the black hole mass $M$ and the mass ratio $q$ of the progenitor binary. In computing the covariance matrix, we used, as described in the previous section, a signal model with 


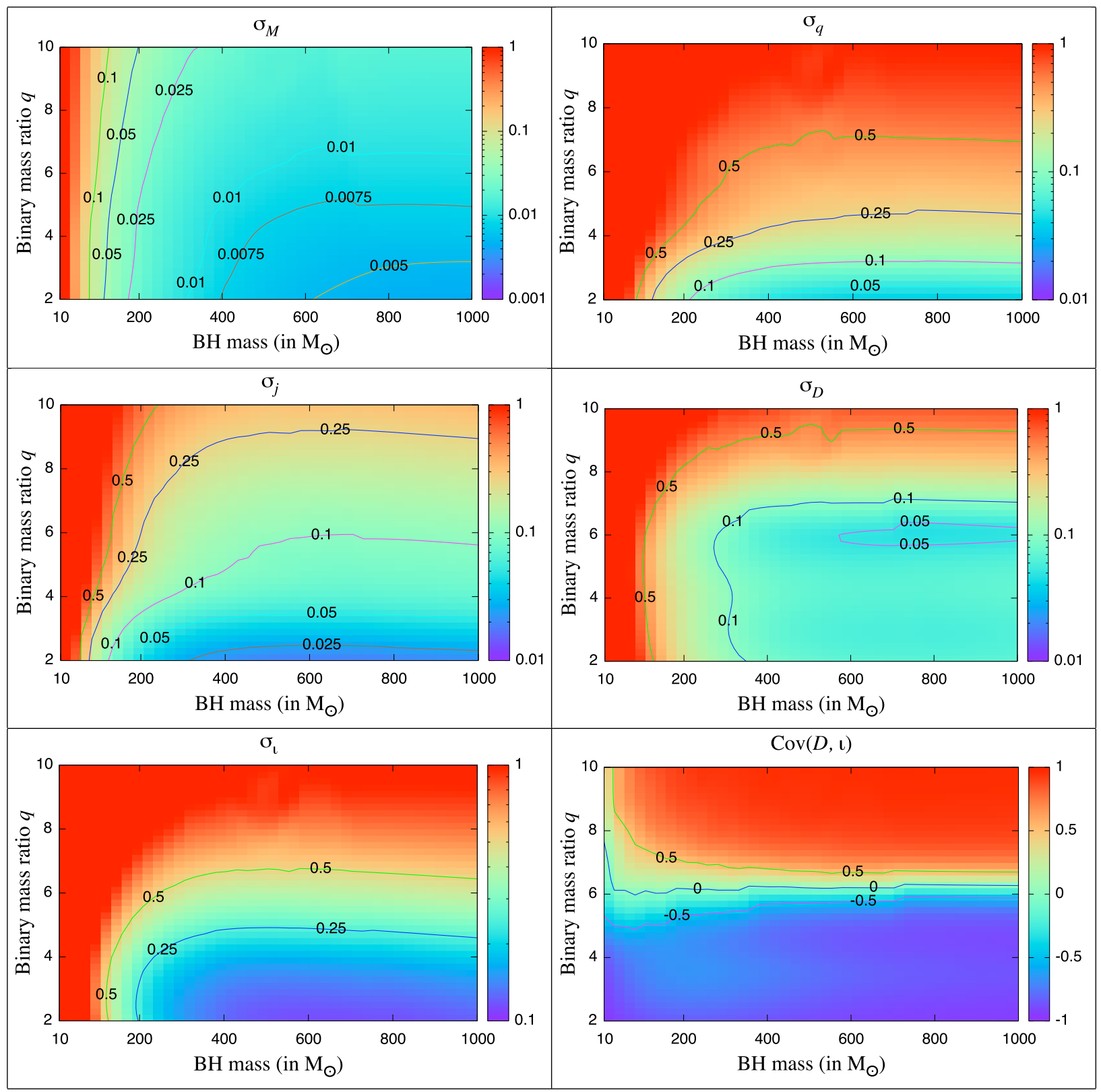

FIG. 7. Dimensionless fractional errors $\sigma_{k} / \lambda_{k}$ in various parameters as a function of the black hole mass and progenitor binary's mass ratio. The black hole is assumed to form at a luminosity distance of $D_{\mathrm{L}}=1 \mathrm{Gpc}$ and the various angles are assumed to be as in Table III. The bottom right panel plots the correlation coefficient between the luminosity distance and the orientation $\iota$ of the black hole's spin with respect to the line-of-sight. The general trend for the errors is to increase with increasing mass ratio and decreasing mass, except for $D_{\mathrm{L}}$ for which there appears to be an 'island' around $q=6$. We have used the ET-B sensitivity curve in computing the covariance matrix.

seven parameters: $\lambda^{k}=\left\{M, j, q, D_{\mathrm{L}}, \iota, \psi, \phi\right\}$. The full covariance matrix contains 7 variances $C_{k k}$ and 21 covariances $C_{m k}=C_{k m}, k \neq m$. The full set of results is too large as our covariance matrix contains 28 independent elements at each point in the $(M, q)$ plane. To save space, we have chosen a subset of these for further discussion.

We will discuss the error in the estimation $\sigma_{k}=\sqrt{C_{k k}}$ of 5 of the 7 parameters, $\lambda^{k}=\left\{M, j, q, D_{\mathrm{L}}, \iota\right\}$, and also include in our discussion the covariance between the luminosity distance $D_{\mathrm{L}}$ and the inclination $\iota$ of the black hole's spin axis with the line-of-sight. Also, it is instructive to deal with the correlation coefficient defined as $c_{k m} \equiv C_{k m} /\left(\sigma_{k} \sigma_{m}\right)$, instead of the covariances themselves. Correlation coefficients are bound to the range $[-1,1]$ and capture how variation in one parameter might be offset by varying another. A correlation coefficient of 0 for a pair of parameters indicates that they are com- 


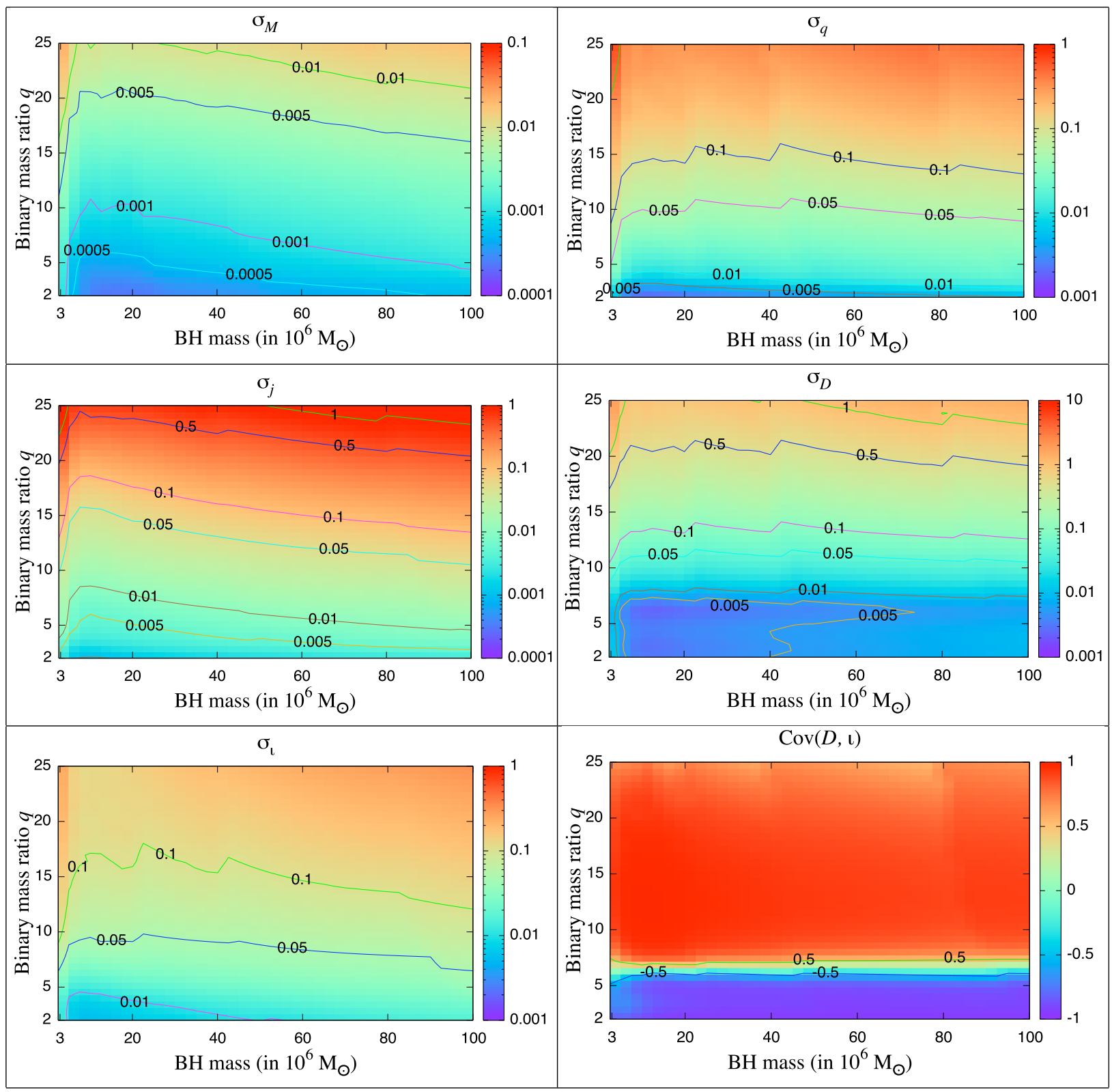

FIG. 8. As in Fig. 7 but for supermassive black holes observed in LISA at a red-shift of $z=1$. Note also that the mass ratio $q$ in this case is allowed to vary from 2 to 25 .

pletely independent of each other and have "orthogonally" different effects on the waveform. For instance, the amplitude $A$ and phase $\varphi$ of a simple sinusoid function $s(t)=A \sin \left[\omega\left(t-t_{0}\right)+\varphi\right]$ will have a correlation coefficient of 0 , while the phase and time offset $t_{0}$ are perfectly anti-correlated and so have a correlation coefficient of -1 : A change in the phase cannot be mimicked by a change in the amplitude but it is completely replicated by a change in the time offset. Consequently, the amplitude is completely independent of the phase and time offset, while only one of phase or time offset can be considered to be an independent parameter 7 . When covariances are close to \pm 1 , the parameters concerned will have large uncertainties and this, as we shall see below, is a major source of error for the parameter $8\left(D_{\mathrm{L}}, \iota\right)$.

Figures 7 and 8 plot fractional errors (i.e. $\sigma_{\lambda_{k}} / \lambda_{k}$ ) incurred in the measurement of the parameters $\left(M, q, j, D_{\mathrm{L}}\right)$ and the absolute error in the parameter $\cos \iota$. We have also plotted the correlation coefficient

\footnotetext{
${ }^{7}$ In fact, the Fisher matrix for the parameter set $\left(A, \varphi, t_{0}\right)$ will, as can easily be verified, be singular.

8 This important point was noted recently by Nissanke et al [70].
} 
$c_{D_{\mathrm{L}} \iota}$, labelled in the figure as $\operatorname{Cov}(D, \iota)$. Figure 7 corresponds to ET's observation of stellar and intermediate mass black holes in the range $[10,1000] M_{\odot}$ and the mass ratio $q$ of the progenitor binary in the range $[2,10]$. Figure 8 corresponds to LISA's observation of supermassive black holes of mass $M$ in the range $\left[3 \times 10^{6}, 10^{8}\right] M_{\odot}$ and mass ratio $q$ in the range $[2,25]$. In the case of aLIGO, in most of the parameter space and for all parameters (except the total mass), the fractional errors are larger than $50 \%$ and so they are not shown. Advanced LIGO will determine the total mass of an intermediate mass black hole that forms from the merger of two nearly equal mass black holes within $1 \mathrm{Gpc}$ to within a few percents and this could be very interesting for some of the tests of general relativity to be discussed below.

Let us recall that our results for mass ratios greater than 11 are based on the extrapolation of analytical fits to numerical simulations of binary black hole mergers that are only available up to a mass ratio of $q=11$. In the next few sections we will discuss our results in the context of the science questions they can address.

\section{A. Mass ratio and component masses of the progenitor binary}

A key result of our study is that one can measure the mass ratio of a progenitor binary by observing the ringdown signals emitted by the black hole that forms from the merger. While the different mode frequencies and time-constants all depend only on the mass of the black hole and its spin magnitude, their relative amplitudes depend on the mass ratio. As we have argued before, under certain circumstances it should be possible to measure the relative amplitudes of the different modes, thereby measure the mass ratio of the progenitor binary and hence deduce its component masses.

The top right panels of Figs. 7 and 8 show the fractional accuracy with which the mass ratio can be determined assuming that the signal is composed of three ringdown modes, namely $(2,2),(3,3)$ and $(2,1)$. ET will not measure the mass ratio very well in most of the parameter space. However, for equal mass mergers, ET should constrain the mass ratio to within 5\%. Remarkably, LISA will be able to measure the mass ratio to better than $10 \%$ over $60 \%$ of the parameter space studied and black holes that result from the merger of equal mass black holes enable the determination of $q$ to better than $1 \%$.

Since the mass ratio of a binary is easily determined from the inspiral phase, its measurement also from the ringdown phase offers newer tests of general relativity.

\section{B. Mass loss to gravitational radiation}

In the process of inspiral and merger, a binary black hole emits a significant fraction (a few percent) of its mass as gravitational radiation. The total mass of a binary can be measured very accurately from the inspiral radiation it emits. Estimates ${ }^{9}$ range from a fraction of a percent (for an equal mass binary black hole of total mass $200 M_{\odot}$ at a distance of $1 \mathrm{Gpc}$ ) in the case of Einstein Telescope [71 to 50 parts per million (for an equal mass binary black hole of total mass $2 \times 10^{6} M_{\odot}$ at a distance of $3 \mathrm{Gpc}$ ) in the case of LISA [26]. For most binaries observed with ET and LISA, the total mass before merger can be measured with an error that is much smaller than the fraction of mass that is expected to be lost in gravitational radiation during merger.

What we see from the top left panels of Figs. 7 and 8 is that the mass of the final black hole that results from a merger can also be measured very accurately. ET cannot measure masses of stellar mass black holes very well but if intermediate mass black holes (and binaries composed of such black holes) exist, then ET will measure their masses to better than $1 \%$, assuming the source is at $1 \mathrm{Gpc}$, over a significant range of the parameter space $(q \lesssim 6$ and $\left.M>400 M_{\odot}\right)$ we explored. A black hole that results from the merger of two black holes each of mass roughly about $500 M_{\odot}$ could be measured to an accuracy of better than half-a-percent.

LISA is able to measure the mass of a supermassive black hole that it observes at a red-shift of $z=1$ with an accuracy of better than $1 \%$ all over the parameter space that we explored. Masses of supermassive black holes that form from the merger of two roughly equal-mass $(q \lesssim 10)$ black holes could be measured to an accuracy of $0.1 \%$. This means that from the ringdown signal alone, we should be able to measure masses of supermassive black holes even at a red-shift of $z=5$ to better than $1 \%$.

It would, therefore, be very interesting to compare the observed mass loss with the predictions of analytical and numerical relativity and verify if the mass loss is in accord with their predictions. Such comparisons will put general relativity to new kinds of tests in the dissipative regime of the theory. Let us recall that the luminosity of a binary black hole, close to merger, could be as large as $10^{50} \mathrm{~J} \mathrm{~s}^{-1}$, which is arguably the largest luminosity any physical system could have. It would be very interesting to test the theory when the luminosity is as large as this.

These phenomenal accuracies with which masses can be measured raise the question if it is prudent to treat the mass of a binary to be constant in the course of its inspiral and merger. It might be possible to deduce the rate of mass loss by treating in our computation of the

\footnotetext{
${ }^{9}$ Note that most of the literature quotes error in the measurement of the chirp mass $\mathcal{M} \equiv M \nu^{3 / 5}$, where $M$ is the total mass and $\nu$ is the symmetric mass ratio of the binary. To estimate the error in the total mass we have used the error propagation formula

$$
\left(\frac{\sigma_{M}}{M}\right)^{2}=\left(\frac{\sigma_{\mathcal{M}}}{\mathcal{M}}\right)^{2}+\frac{9}{25}\left(\frac{\sigma_{\nu}}{\nu}\right)^{2}-\frac{6}{5} c_{\mathcal{M}, \nu}\left(\frac{\sigma_{\mathcal{M}}}{\mathcal{M}}\right)\left(\frac{\sigma_{\nu}}{\nu}\right) .
$$

where $c_{\mathcal{M}, \nu}$ is the correlation coefficient of the parameters $\ln \mathcal{M}$ and $\ln \nu$.
} 
waveforms the mass of the system to be a function of time.

\section{Exploring naked singularities}

The magnitude and orientation of the spin of the final black hole that results from the merger of a black hole binary depend on a number of parameters of the progenitor binary: magnitudes and orientations of the spins of the two component black holes relative to the orbital angular momentum and the mass ratio of the progenitor binary. A spinning black hole binary has a rather large parameter space, six parameters more than a non-spinning system. Limited studies have been carried out in assessing how well one might be able to measure black hole spins from the inspiral phase of the merger of a black hole binary [72, 73]. Numerical relativity simulations of the merger of spinning black holes are still in their early stages. In the coming years we are likely to learn a great deal about spin dynamics of a binary before and after merger. While these are important problems to be addressed in the future, we recall that in this paper we have only studied binaries comprising of initially non-spinning black holes.

Figures 7 and 8, middle left panels, show how accurately one might be able to measure the spin magnitude $j$ in ET and LISA, respectively. The accuracy here is not as good as in the case of the black hole mass. ET can deduce the final spin to within $10 \%$ over $40 \%$ of the parameter space and to better than $5 \%$ in $20 \%$ of the parameter space, for black holes that form within $1 \mathrm{Gpc}$. LISA, on the other hand, can measure spin magnitudes to better than $5 \%$ over $50 \%$ of the parameter space and to better than $1 \%$ over $20 \%$ of the parameter space for black holes that form within a red-shift of $z=1$. Spins of black holes that form from the merger of nearly equal mass black holes can be measured to $0.5 \%$. Thus, LISA should be able to reliably measure spin magnitudes that are only a few percent larger than 1. Measuring spin magnitudes to such a high accuracy will be useful in testing whether a merging binary results in a black hole or a naked singularity [17.

\section{Spin orientations and the luminosity distance}

The measurement accuracy of spin orientation, given by $\cos \iota$, is shown in the bottom left panels of Figs.7] and 8. $\cos \iota$ can be measured to within $10 \%$ in about onethird of the parameter space in the case of ET and to better than $5 \%$ in about one-half of the parameter space in the case of LISA. The dynamics of spins before and after merger could be relevant in understanding the $\mathrm{x}$ shaped radio galaxies [74].

The spin orientation of a black hole is very strongly correlated with the luminosity distance. We see from the bottom right panels that the correlation coefficient is close to either +1 or -1 in most of the parameter space.
The transition from negative to positive correlation between $D_{\mathrm{L}}$ and $\cos \iota$ occurs when mass ratio $q \simeq 6$. The significance of this number is not clear to us at the moment. This correlation completely destroys the accuracy with which the luminosity distance can be measured. For instance, we see that in the case of ET $\sigma_{D_{\mathrm{L}}} / D_{\mathrm{L}}$ is in the range $5-10 \%$, although the $\mathrm{SNR}$ in this region of the parameter space is $\sim 300$. The parameter $\cos \iota$ in this region is also determined rather poorly, at about $25 \%$.

These numbers are better in the case of LISA: For $50 \%$ of the parameter space, LISA can measure the distance to within $10 \%$ but black holes that result from the merger of roughly equal mass systems might allow the luminosity distance to be determined to $1 \%$ or less. Using the inspiral phase alone, but with the help of sub-dominant signal harmonics, luminosity distance can be measured to a fraction of a percent in the case of LISA. Thus, it would be very interesting to see how similar are the distances obtained from these different phases of the merger dynamics.

\section{PARAMETRIZATION FOR TESTING THE NO-HAIR THEOREM}

In this section we will consider a practical implementation of testing general relativity using quasi normal modes. To test general relativity with quasi-normal modes, it is not necessary to consider all the physical parameters but only those that are necessary to fully characterize the shape of the signal. The ringdown signal composed of a superposition of $n$ quasi-normal modes can be written as

$$
h(t)=\sum_{\ell, m} A_{\ell m} e^{-t / \tau_{\ell m}} \cos \left(\omega_{\ell m} t+\gamma_{\ell m}\right),
$$

where there are $n$ each of the amplitudes $A_{\ell m}$, timeconstants $\tau_{\ell m}$, frequencies $\omega_{\ell m}$ and phases $\gamma_{\ell m}$. The signal is, therefore, characterized by a set of $4 n$ parameters in total.

\section{A. Maximal set}

The most exhaustive test of the no-hair theorem would be to treat all $4 n$ parameters to be independent. Of the $4 n$ parameters, only the $2 n$ mode frequencies and timeconstants would facilitate the test, the others should be retained in order to fully capture the covariances and variances in the $2 n$ test parameters. The consistency among every mode frequency and time constant makes the test more stringent but the presence of a large number of parameters (when $n$ is greater than 2) weakens the test. The reason for the latter is that a model with too many parameters will/should be penalized for its flexibility by any carefully formulated test. In a covariance matrix formulation of the test, this will be reflected by 

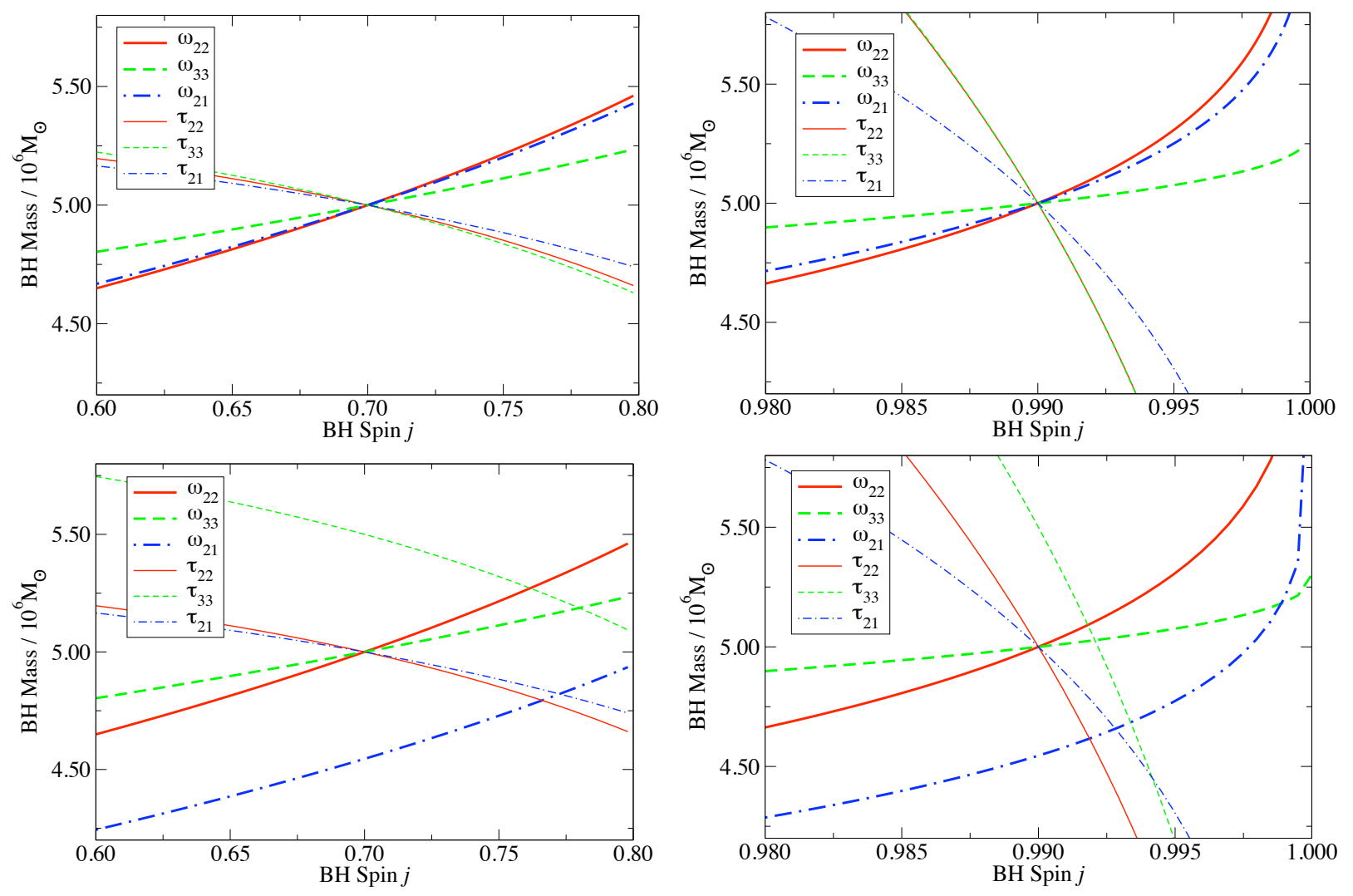

FIG. 9. Curves of constant mode frequencies and time-constants in the $(M, j)$-plane obtained with Eqs. 43 for the $(2,2)$ mode and similar equations for other modes. Top panels correspond to the case when all measured values are exactly as predicted by black hole perturbation theory. The bottom panels correspond to the case where the $(3,3)$ and $(2,1)$ mode frequencies and time constants differ from the GR value by $10 \%$ but the $(2,2)$ frequency and time-constant are as in GR. An interesting thing to note is that for a large range of values of the black hole spin, the curves of constant $\tau_{22}$ and $\tau_{33}$ almost overlap, providing the opportunity for a more accurate test of the no-hair theorem. (see the text for details.)

large variances in $\tau_{\ell m}$ and $\omega_{\ell m}$ (which are our test parameters) and in a Bayesian model selection a model with a larger number of parameters will suffer from having a large evidence.

How would the test work in practice? For each measured test parameter (and the associated error in its measurement), one could draw a curve (or a band including the error) in the $(M, j)$ plane, by using their expressions in general relativity in terms of the mass and spin of the black hole. If the curves/bands fail to intersect at a single point/region in the $(M, j)$ plane then that would invalidate general relativity or, alternatively, indicate that the object is not a black hole. The maximal set could be weak due to the large variances of the various parameters but strong because many different bands have to pass through the same region.

Let us illustrate how the test works with some examples. Let us suppose our signal model consists of a superposition of $(\ell, m)=(2,2),(2,1),(3,3),(4,4)$ modes. In this case the maximal set contains 16 parameters, of which 8 mode frequencies and time-constants are the test parameters. Let us denote by $\hat{\omega}_{\ell m}$ and $\hat{\tau}_{\ell m}$, the values of the mode frequencies and time-constants measured by projecting the data onto a superposition of quasi-normal modes as in Eq. 42. For each measured parameter, we can construct an equation in $(M, j)$ using the relation between the parameter and $(M, j)$ given in Eqs. 27)-(30):

$$
\begin{aligned}
& \hat{\omega}_{22}=\frac{1}{M}\left[1.5251-1.1568(1-j)^{0.1292}\right], \\
& \hat{\tau}_{22}=\frac{2}{\omega_{22}}\left[0.7000+1.4187(1-j)^{-0.4990}\right],
\end{aligned}
$$

and similar equations for other modes. (Note that we have used $f_{22}=M \omega_{22}$ and $2 Q_{\ell m}=\tau_{\ell m} \omega_{\ell m}$ in rewriting these equations as dimensionful quantities.) Measurement errors can be folded into the analysis by using

$$
\begin{aligned}
\hat{\omega}_{22} \pm \sigma_{\omega_{22}} & =\frac{1}{M}\left[1.5251-1.1568(1-j)^{0.1292}\right], \\
\hat{\tau}_{22} \pm \sigma_{\tau_{22}} & =\frac{2}{\omega_{22}}\left[0.7000+1.4187(1-j)^{-0.4990}\right],
\end{aligned}
$$

with similar equations for other modes.

For a $5 \times 10^{6} M_{\odot}$ black hole, the mode frequencies and time-constants for three different spin values $j=$ 
$0.1,0.7,0.9,0.99$, are given in Table [V] If the ringdown

TABLE IV. The frequencies $F_{\ell m}=\omega_{\ell m} /(2 \pi)$ (in $\mathrm{mHz}$ ) and time-constants $\tau_{\ell m}$ (in s) of the first four dominant modes for a $5 \times 10^{6} M_{\odot}$ black hole of different spin magnitudes $j$.

\begin{tabular}{rrrrrrrrr}
\hline \hline$j$ & $F_{22}$ & $F_{21}$ & $F_{33}$ & $F_{44}$ & $\tau_{22}$ & $\tau_{21}$ & $\tau_{33}$ & $\tau_{44}$ \\
\hline 0.10 & 2.48 & 2.43 & 3.98 & 5.36 & 282 & 277 & 269 & 266 \\
\hline 0.70 & 3.46 & 2.96 & 5.48 & 7.44 & 303 & 301 & 295 & 290 \\
\hline 0.90 & 4.30 & 3.30 & 6.70 & 9.06 & 383 & 355 & 379 & 375 \\
\hline 0.99 & 5.73 & 3.66 & 8.60 & 11.4 & 823 & 559 & 828 & 837 \\
\hline \hline
\end{tabular}

signal is consistent with the formation of a black hole with spin magnitude, say, $j=0.7(j=0.99)$ then the mode frequencies and time-constants would be precisely as in the 2nd (respectively, 4th) row of Table IV modulo the errors in their measurement. Therefore, curves defined by $\omega_{\ell m}(M, j)=\hat{\omega}_{\ell m}$ and $\tau_{\ell m}(M, j)=\hat{\tau}_{\ell m}$ will all meet at a single point in the $(M, j)$ plane as in the upper two panels of Fig. 9 , the point of intersection giving the mass and spin magnitude of the black hole. The left panel corresponds to the formation of a black hole of spin magnitude $j=0.7$ and the right panel to $j=0.99$, in both cases $M=5 \times 10^{6} M_{\odot}$. If, however, one of the mode frequencies, say $\omega_{33}$ is different from the general relativistic value by $10 \%$, then the corresponding curves would fail to meet as shown in the lower two panels of Fig. 9

If general relativity is true then some of the curves lie almost one on top of the other (e.g. $\omega_{22}=$ const. is almost identical to $\omega_{21}=$ const. in the $j=0.7$ case and $\tau_{22}=$ const. is identical to $\tau_{33}=$ const. both in the $j=0.7$ and $j=0.99$ cases) but even a slight departure from general relativity will lead to big departures as demonstrated by the lower panels of the same figure. In any measurement the parameters are subject to statistical and systematic errors that must be folded into the analysis which will be taken up in a forthcoming study.

Do we need to treat all time-constants and mode frequencies to be independent in a test of the no-hair theorem? We shall argue below that it is not necessary to treat all $4 n$ parameters to be independent; in fact, we shall see that the parametrization is not unique, offering a lot of flexibility in testing GR.

\section{B. Minimal set}

The minimal, or the simplest, model would consist of the smallest number of parameters needed to check the consistency between the modes as predicted by the nohair theorem, yet large enough to capture all the variances and covariances between the parameters of interest. Since the mode frequencies and time-constants are all determined in GR by the mass $M$ and spin magnitude $j$ of the black hole, the smallest number of parameters required to test GR would be three: these could be any three time-constants or two time-constants and one mode frequency, and so on. It would then be necessary to express the other mode frequencies and time-constants in terms of any two of the three parameters that were taken to be independent. Two of the three independent parameters could be used to solve for $(M, j)$. One could then see if the measured value of the third parameter is consistent with its predicted value based on the values of $M$ and $j$. Let us note that without a prior knowledge of the amplitudes $A_{\ell m}$ and phases $\gamma_{\ell m}$ it will not be possible to measure the chosen time-constants and mode frequencies as they would induce covariances that cannot be neglected in estimating the errors incurred in their measurement. Thus, we (tentatively) conclude that the minimal set required for a signal model with $n$ modes would be $2 n+3$. However, the three test parameters can be chosen in any way one wishes but choosing only three assures that the error in their measurement is the smallest. In this sense the minimal set could be a very stringent test of GR.

In reality, of course, the amplitudes of different modes are determined by the physical parameters $\left(M, j, q, D_{\mathrm{L}}, \theta, \varphi, \psi, \iota\right)$ (cf. the discussion at the beginning of this section). We can assume the parameters $\left(q, D_{\mathrm{L}}, \theta, \varphi\right)$ to be known from the inspiral phase and $(M, j)$ to be determined by the time-constants and/or mode frequencies of the ringdown signal. This leaves the two angles $(\psi, \iota)$. Thus, when $n$ is greater than 2 , it is not necessary to consider all mode amplitudes to be independent but just two of them. Thus the minimal set of parameters to be considered for testing the no-hair theorem is $n+5$ ( $n$ phases, $\gamma_{\ell m}$, the 3 test parameters and the two angles $\psi$ and $\iota)$.

\section{CONCLUSIONS AND FUTURE WORK}

In this paper, we have explored what information can be extracted from a black hole's ringdown signal, wherein the perturbation is caused by the tidal deformation produced during the merger of two non-spinning black holes. To this end we used numerical simulations of the late inspiral and ringdown to estimate the relative amplitudes of the various modes excited. The simulations consisted of initially non-spinning black holes in quasi-circular orbits for several mass ratios, ranging from 1:1 to 1:11.

We find that several modes have large enough luminosity - or signal-to-noise ratio - to be detectable in LISA and ET. Specifically, in order of decreasing power, modes $(2,2),(3,3),(2,1),(3,2),(4,4),(5,5),(4,3),(6,6)$, $(5,4)$ and $(4,2)$ for LISA and the first four to five modes for ET, have significant luminosities. Note that not all of these can necessarily be resolved, but it probably is the case for the first three (see below). In the analysis though, we decided to include only $(2,2),(3,3)$ and $(2,1)$, mainly because the available data were most accurate for those modes.

We argued that the ringdown signal depends on the mass ratio of the progenitor binary and that this can be 
measured, with an error that is estimated from a Fisher matrix analysis. We showed how the luminosities change with the mass ratio. Indeed, by constructing fits to the mode amplitudes in terms of the mass ratio, we were able to include this effect in the analysis and estimate the errors involved in the measurement of various parameters. An important issue was to determine the epoch when the ringdown phase starts, so as to evaluate the relative amplitudes at that point. This was taken to be the point where each mode's frequency stops having an upward trend. The epoch at which the peak luminosity is reached is slightly different for different modes, but the mode amplitudes were all measured at a time $10 M$ after the peak luminosity of the 22 mode.

We computed the measurement errors of a number of other parameters for ringdowns observed in LISA and ET. These include the black hole mass, its spin, luminosity distance and inclination angle and how these vary with the final $\mathrm{BH}$ mass and the binary mass ratio. If we do not consider initial BH spins, the inclination angle can be measured solely by observing the three most dominant modes of the ringdown waveform, assuming that these can be resolved.

Together, LISA and ET will be able to provide ample evidence for the distribution of supermassive, intermediate mass and stellar mass black holes, for a large part of the known Universe. For most of the parameter space, the reach of LISA for ringdowns is $z \sim 6$, while for ET at least $z \sim 0.8$. Also, by being able to measure the mass ratio, hints on the merger history and formation of black holes of a large range of masses could be inferred by studying ringdown signals.

A practical implementation for testing the no-hair theorem and deciding the nature of the compact object that results from the merger, was presented, illustrating several key components. We started by providing a general framework, which is based on the number of parameters necessary to apply the test. Specifically, using $n$ modes, a generic test will use the $2 n$ frequencies and timeconstants. This test is implemented by plotting the $\omega_{\ell m}$ and $\tau_{\ell m}$ curves on the mass-spin plane of the final black hole, where all the curves should intersect inside the same region if the object is a black hole. A key point was that some of the curves are special, as they almost overlap and can be thus used to check for small deviations from general relativity.

Future work should extend the study to include numerical simulations of initially spinning black holes, as this corresponds to a more realistic scenario. Additionally, there is effort to produce more accurate numerical simulations so that less dominant modes could be studied when the mass ratio is large.

The current study has not investigated the question of decomposition of modes of a ringdown signal in real data. The SNRs of different modes, especially in the case of LISA, suggest that it should be possible to resolve the modes and carry out the proposed tests of general relativity. However, a more in-depth investigation needs to be done, for instance by using a Bayesian model selection to discriminate between different models. Given some prior information, the relative probability of two different multimode ringdown waveforms - injected in Gaussian and stationary noisy data - could be computed.

Finally, a Fisher matrix-based parameter estimation approach (such as the one presented in this work) may not be robust or accurate enough, especially when the parameter space is large and the signal-to-noise ratios are low 75]. For this reason, future studies should also involve parameter estimation in the context of the afore mentioned Bayesian analysis, as well as a Bayesian approach to a test of the no-hair theorem. In the latter case the posterior joint probabilities of two different models, given the 'initial data', could be compared. The first hypothesis would be that the observed object is a black hole, while the second that either GR is incorrect or the merged object is not a black hole.

\section{ACKNOWLEDGMENTS}

We are grateful to Alessandra Buonanno, Vitor Cardoso, Thomas Dent, Ajith Parameswaran and Evan Ochsner for a careful reading of the manuscript and comments that helped improve parts of the paper. BSS was funded by the Science and Technology Facilities Council (STFC) Grant No. ST/J000345/1 and MH by STFC Grant No. ST/H008438/1.
[1] R. Kerr, Phys. Rev. Lett. 11, 237 (1963)

[2] E. Newman, K. Couch, K. Chinnapared, A. Exton, A. Parakash, and Torrence, J. Math. Phys. 6, 918 (1965)

[3] W. Israel, Phys. Rev. 164, 1776 (1967)

[4] W. Israel, Commun. Math. Phys. 8, 245 (1968)

[5] B. Carter, Phys. Rev. Lett. 26, 331 (1971)

[6] S. Hawking, Commun. Math. Phys. 25, 152 (1972)

[7] D. Robinson, Phys. Rev. Lett. 34, 905 (1975)

[8] C. Vishveshwara, Nature 227, 936 (1970)

[9] T. Regge and J. Wheeler, Phys. Rev. 108, 1063 (1957)

[10] C. Vishveshwara, Phys. Rev. D 1, 2870 (1970)
[11] W. Press, Astrophys. J. Lett. 170, L105 (1971)

[12] S. A. Teukolsky, Phys. Rev. Lett. 29, 1114 (1972)

[13] S. Teukolsky, Astrophys. J. 185, 635 (1973)

[14] W. Press and S. Teukolsky, Astrophys. J. 185, 649 (1973)

[15] B. F. Whiting, Journal of Mathematical Physics 30, 1301 (Jun. 1989)

[16] E. Berti, V. Cardoso, and A. O. Starinets, Class. Quant. Grav. 26, 163001 (2009), arXiv:0905.2975 [gr-qc]

[17] O. Dreyer, B. Kelly, B. Krishnan, L. S. Finn, D. Garrison, and R. Lopez-Aleman, Class. Quantum Grav. 21, 787 (2004), gr-qc/0309007 
[18] E. Berti, V. Cardoso, and C. M. Will, Phys. Rev. D 73 , 064030 (Mar. 2006), gr-qc/0512160

[19] E. Berti, J. Cardoso, V. Cardoso, and M. Cavagliá, Phys. Rev. D76, 104044 (2007), arXiv:0707.1202

[20] K. Danzmann, Class. Quantum Grav. 14, 1399 (1997)

[21] M. Punturo, M. Abernathy, F. Acernese, B. Allen, N. Andersson, et al., Class.Quant.Grav. 27, 194002 (2010)

[22] D. Shoemaker, Advanced LIGO Anticipated Sensitivity Curves, Tech. Rep. (2009) https://dcc.ligo.org/ cgi-bin/DocDB/ShowDocument?docid=2974

[23] J. Smith (for the LIGO Scientific Collaboration), Classical and Quantum Gravity 26, 114013 (Jun. 2009)

[24] G. M. Harry and the LIGO Scientific Collaboration, Classical and Quantum Gravity 27, 084006 (2010)

[25] B. Abbott et al., Advanced LIGO Reference Design, Tech. Rep. LIGO-M060056-08-M (LIGO Project, 2007) http://www.ligo.caltech.edu/docs/M/M060056-08/ M060056-08.pdf

[26] K. G. Arun, Phys. Rev. D 74, 024025 (Jul. 2006), grqc/0605021

[27] P. Amaro-Seoane and L. Santamaria, Astrophys.J. 722, 1197 (2010), arXiv:0910.0254 [astro-ph.CO]

[28] B. Brügmann et al., Phys. Rev. D77, 024027 (2008), arXiv:gr-qc/0610128

[29] S. Husa, J. A. González, M. Hannam, B. Brügmann, and U. Sperhake, Class. Quant. Grav. 25, 105006 (2008), arXiv:0706.0740 [gr-qc]

[30] S. Brandt and B. Brügmann, Phys. Rev. Lett. 78, 3606 (1997), arXiv:gr-qc/9703066

[31] J. M. Bowen and J. W. York, Jr., Phys. Rev. D21, 2047 (1980)

[32] M. Ansorg, B. Brügmann, and W. Tichy, Phys. Rev. D70, 064011 (2004), arXiv:gr-qc/0404056

[33] M. Campanelli, C. O. Lousto, P. Marronetti, and Y. Zlochower, Phys. Rev. Lett. 96, 111101 (2006), arXiv:grqc/0511048

[34] J. G. Baker, J. Centrella, D.-I. Choi, M. Koppitz, and J. van Meter, Phys. Rev. Lett. 96, 111102 (2006), arXiv:gr-qc/0511103

[35] M. Hannam, S. Husa, D. Pollney, B. Brügmann, and N. Ó Murchadha, Phys. Rev. Lett. 99, 241102 (2007), arXiv:gr-qc/0606099

[36] M. Shibata and T. Nakamura, Phys. Rev. D52, 5428 (1995)

[37] T. W. Baumgarte and S. L. Shapiro, Phys. Rev. D59, 024007 (1999), arXiv:gr-qc/9810065

[38] S. Husa, M. Hannam, J. A. González, U. Sperhake, and B. Brügmann, Phys. Rev. D77, 044037 (2008), arXiv:0706.0904 [gr-qc]

[39] M. Hannam, S. Husa, F. Ohme, D. Muller, and B. Bruegmann, Phys.Rev. D82, 124008 (2010), arXiv:1007.4789 $[\mathrm{gr}-\mathrm{qc}]$

[40] E. Berti, V. Cardoso, J. Gonzalez, U. Sperhake, M. Hannam, S. Husa, and B. Brugmann, Phys. Rev. D76, 064034 (2007), arXiv:gr-qc/0703053v2

[41] T. Damour, A. Nagar, M. Hannam, S. Husa, and B. Bruegmann, Phys.Rev. D78, 044039 (2008), arXiv:0803.3162 [gr-qc]

[42] C. Reisswig and D. Pollney, Class.Quant.Grav. 28, 195015 (2011), arXiv:1006.1632 [gr-qc]

[43] M. Hannam, S. Husa, U. Sperhake, B. Bruegmann, and J. A. Gonzalez, Phys.Rev. D77, 044020 (2008), arXiv:0706.1305 [gr-qc]
[44] S. Babak, M. Hannam, S. Husa, and B. F. Schutz(2008), arXiv:0806.1591 [gr-qc]

[45] J. A. Gonzalez, U. Sperhake, and B. Bruegmann, Phys.Rev. D79, 124006 (2009), arXiv:0811.3952 [gr-qc]

[46] C. O. Lousto, H. Nakano, Y. Zlochower, and M. Campanelli, Phys.Rev.Lett. 104, 211101 (2010), arXiv:1001.2316 [gr-qc]

[47] C. O. Lousto, H. Nakano, Y. Zlochower, and M. Campanelli, Phys.Rev. D82, 104057 (2010), arXiv:1008.4360 $[\mathrm{gr}-\mathrm{qc}]$

[48] L. Kidder, Phys. Rev. D 77, 044016 (2008)

[49] D. Pollney and C. Reisswig(2010), arXiv:1004.4209 [grqc]

[50] J. Baker, A. Abrahams, P. Anninos, S. Brandt, R. Price, J. Pullin, and E. Seidel, Phys. Rev. D 55, 829 (Jan. 1997), arXiv:gr-qc/9608064

[51] Y. Pan, A. Buonanno, M. Boyle, L. T. Buchman, L. E. Kidder, et al.(2011), arXiv:1106.1021 [gr-qc]

[52] J. G. Baker, W. D. Boggs, J. Centrella, B. J. Kelly, S. T. McWilliams, and J. R. van Meter, Phys.Rev. D78, 044046 (2008), arXiv:0805.1428 [gr-qc]

[53] J. G. Baker, S. T. McWilliams, J. R. van Meter, J. Centrella, D.-I. Choi, et al., Phys.Rev. D75, 124024 (2007), arXiv:gr-qc/0612117 [gr-qc]

[54] A. Buonanno, L. E. Kidder, and L. Lehner, Phys.Rev. D77, 026004 (2008), arXiv:0709.3839 [astro-ph]

[55] L. Rezzolla, E. Barausse, E. Dorband, D. Pollney, C. Reisswig, J. Seiler, and S. Husa, Phys. Rev. D 78, 044002 (2007), doi:"bibinfo doi 10.1103/PhysRevD.78.044002, arXiv:0712.3541

[56] L. Rezzolla, E. Dorband, C. Reisswig, P. Diener, D. Pollney, E. Schnetter, and B. Szilágyi, Astrophys. J. 679, 1422 (2007), arXiv:0708.3999

[57] L. Rezzolla, P. Diener, E. Dorband, D. Pollney, C. Reisswig, E. Schnetter, and J. Seiler, Astrophys. J. Lett. 674, L29 (2008), arXiv:0710.3345

[58] E. Berti, V. Cardoso, J. Gonzalez, U. Sperhake, and B. Bruegmann, Class.Quant.Grav. 25, 114035 (2008), arXiv:0711.1097 [gr-qc]

[59] K. S. Thorne, in Three hundred years of gravitation, edited by S. Hawking and W. Israel (Cambridge University Press, 1987) pp. 330-458

[60] S. Hild, S. Chelkowski, A. Freise, J. Franc, N. Morgado, et al., Class.Quant.Grav. 27, 015003 (2010), arXiv:0906.2655 [gr-qc]

[61] K. Arun, S. Babak, E. Berti, N. Cornish, C. Cutler, et al., Class.Quant.Grav. 26, 094027 (2009), arXiv:0811.1011 [gr-qc]

62] K. Arnaud, S. Babak, J. Baker, M. Benacquista, N. Cornish, et al., Class.Quant.Grav. 24, S551 (2007), arXiv:grqc/0701170 [gr-qc]

63] S. Babak et al. (Mock LISA Data Challenge Task Force), Class.Quant.Grav. 25, 114037 (2008), arXiv:0711.2667 [gr-qc]

[64] I. Kamaretsos(2011), arXiv:1112.3077 [gr-qc]

[65] L. A. Wainstein and V. D. Zubakov, Extraction of Signals from Noise (Prentice-Hall, Englewood Cliffs, 1962)

[66] L. S. Finn, Phys. Rev. D 46, 5236 (1992)

[67] R. Balasubramanian, B. S. Sathyaprakash, and S. V. Dhurandhar, Phys. Rev. D 53, 3033 (1996), erratumibid. D 54, 1860 (1996), gr-qc/9508011

[68] C. Cutler, Phys. Rev. D 57, 7089 (1998)

[69] K. G. Arun, B. R. Iyer, B. S. Sathyaprakash, S. Sinha, and C. Van Den Broeck, Phys. Rev. D 76, 104016 (2007), 
arXiv:0707.3920

[70] S. Nissanke, D. E. Holz, S. A. Hughes, N. Dalal, and J. L. Sievers, Astrophys.J. 725, 496 (2010), arXiv:0904.1017 [astro-ph.CO]

[71] C. Van Den Broeck and A. S. Sengupta, Class. Quantum Grav. 24, 1089 (Mar. 2007), gr-qc/0610126
[72] A. Vecchio, Phys. Rev. D 70, 042001 (2004)

[73] R. N. Lang and S. A. Hughes, Phys. Rev. D 74, 122001 (2006), erratum-ibid. D 75, 089902 (2007), gr-qc/0608062

[74] D. Merritt and R. Ekers, Science 297, 1310 (2002), astro$\mathrm{ph} / 0208001$

[75] M. Vallisneri, Phys. Rev. D 77, 042001 (2008), grqc/0703086 